\title{
Multiferroic heterostructures for spintronics
}

\section{Review Article}

Author(s):

Gradauskaite, Elzbieta; Meisenheimer, Peter; Müller, Marvin; Heron, John; Trassin, Morgan (D)

Publication date:

2021-02

Permanent link:

https://doi.org/10.3929/ethz-b-000458163

Rights / license:

In Copyright - Non-Commercial Use Permitted

Originally published in:

Physical Sciences Reviews 6(2), https://doi.org/10.1515/psr-2019-0072

Funding acknowledgement:

188414 - Multifunctional oxide electronics using natural ferroelectric superlattices (SNF) 


\title{
This is the pre-peer reviewed version of the following article: Multiferroic heterostructures for spintronics, Phys. Sci. Rev. 2019, which has been published in final form at DOI:10.1515/psr-2019-0072.
}

\section{Multiferroic heterostructures for spintronics}

\author{
Elzbieta Gradauskaite ${ }^{1^{*}}$, Peter Meisenheimer ${ }^{2^{*}}$, Marvin Müller ${ }^{1}$, John Heron ${ }^{2^{*}}$ and Morgan Trassin ${ }^{1^{*}}$ \\ 1 - Department of Materials, ETH Zurich, Vladimir-Prelog-Weg 4, 8093 Zurich, Switzerland \\ 2 - Department of Materials Science and Engineering, University of Michigan, Ann Arbor, Michigan \\ 48109, USA \\ *E-mails: elzbieta.gradauskaite@mat.ethz.ch, meisep@umich.edu, itheron@umich.edu, \\ morgan.trassin@mat.ethz.ch
}

\begin{abstract}
For next generation technology, magnetic systems are of interest due to the natural ability to store information and, through spin transport, propagate this information for logic functions. Controlling the magnetization state through currents has proven energy inefficient. Multiferroic thin film heterostructures, combining ferroelectric and ferromagnetic orders, hold promise for energy efficient electronics. The electric field control of magnetic order is expected to reduce energy dissipation by 2-3 orders of magnitude relative to the current state-of-the-art. The coupling between electrical and magnetic orders in multiferroic and magnetoelectric thin film heterostructures relies on interfacial coupling though magnetic exchange or mechanical strain and the correlation between domains in adjacent functional ferroic layers. Here we review the recent development dealing with electrical control of magnetism in artificial magnetoelectric heterostructures, domain imprint, emergent physics and device paradigms for magnetoelectric logic, neuromorphic devices, and hybrid magnetoelectric/spin-current based applications. Finally, we conclude with discussing experiments that probe the crucial dynamics of the magnetoelectric switching and optical tuning of ferroelectric states towards all optical control of magnetoelectric switching events.
\end{abstract}

Keywords: multiferroics, $\mathrm{BiFeO}_{3}, \mathrm{Cr}_{2} \mathrm{O}_{3}$, magnetoelectrics, artificial multiferroics, spintronics, ferroic domain imprint, exchange bias, magnetostriction, memory and logic 


\section{Introduction}

\section{1 Magnetoelectric multiferroic heterostructures}

Multiferroic materials, exhibiting multiple order parameters in a single-phase, were first discussed in the 50's [1] and have been in the recent years at the center of interest with the development of experimental techniques for the design and probe of such complex materials [2,3]. Here, we will consider materials exhibiting ferroelectricity and ferromagnetism for spintronic applications [4,5]. When the two orders coexist but are not coupled, so-called multiferroic memory elements can be envisioned [6]. In such memories, the information can be stored independently in each order parameter, increasing the storage density in a given active space. The focus of the scientific community, however, is shifting towards magnetoelectric multiferroic materials, where the magnetic and electric orders are coupled, meaning that a magnetic state can be electrically altered. Magnetoelectric-based device paradigms [7] could solve the current limitations accompanying the magnetic data storage development and the energy costs associated with magnetization reversal, as state-of-the-art magnetization switching processes involve high-density spin polarized currents, which result in extreme heat dissipation and limited energy efficiency. Using an electric field to reverse a magnetic bit could lead to current-less and extremely localized switching events through voltage application to a capacitor.

Technologically relevant magnetoelectric multiferroic materials, i.e. those possessing coexisting and robustly coupled magnetization and ferroelectric polarization at elevated temperature, are scarce. The conditions for the establishment of a polarization and magnetization in a material are often mutually exclusive [8], yet, several mechanisms allow for a polarization to coexist with magnetic order [1]. For example, improper ferroelectric materials, in which the polarization is not the primary order parameter, often exhibit magnetic order. Sometimes the magnetism itself can trigger a polarization, causing an induced ferroelectricity to emerge at low temperature. In this case, however, the polarization values are too small for device integration [1]. Most single-phase multiferroic magnetoelectric materials exhibit a ferroelectric polarization in an antiferromagnetic matrix, the prototypical materials being $\mathrm{YMnO}_{3}$ and

$\mathrm{BiFeO}_{3}$. We emphasize here that $\mathrm{Cr}_{2} \mathrm{O}_{3}$, discussed in the following sections is not multiferroic, however, its established magnetoelectric effect at room temperature $[9,10]$ brings this material in the focus regarding magnetoelectric-based device research. Hexagonal $\mathrm{YMnO}_{3}$ belongs to the improper ferroelectric category, in which the ferroelectricity emerges a consequence of steric effects [1,11], it is also antiferromagnetic below 130 K $[1,12,13]$. The integration of $\mathrm{YMnO}_{3}$ into thin film heterostructures and the accompanying impact of epitaxial interface on the exotic ferroelectric state has only been investigated recently [14]. Most 
of the multiferroic and application relevant advances in hexagonal manganites are dealing with bulk measurements [15-17].

In contrast, $\mathrm{BiFeO}_{3}$ is the most studied multiferroic thin-film material $[1,18]$. Its room temperature ferroelectricity with extremely high polarization values, reaching up to $150 \mu \mathrm{C} \mathrm{cm}^{-2}$ [19], and G-type antiferromagnetic order $[20,21]$ offer a unique platform to study the potential applications of multiferroic magnetoelectric materials. We recommend recent reviews dedicated to the material for further reading $[22,23]$.

With the progress in growth techniques, new multiferroic magnetoelectric candidates have emerged as alternatives to $\mathrm{BiFeO}_{3}$-based heterostructures. For instance, $\mathrm{GaFeO}_{3}$ [24-26], exhibiting a higher magnetoelectric coefficient than the reference $\mathrm{Cr}_{2} \mathrm{O}_{3}$ material, can now be deposited as high quality thin films [27]. Similarly, layered materials such as Aurivillius phases $[28,29]$, only recently grown in a single-crystalline epitaxial form [30], show robust ferroelectric properties in the ultrathin regime [30-33] in a structure that is compatible with long-range magnetic order $[34,35]$.

In order to compensate for the current lack of materials exhibiting strong and coupled ferroelectric and magnetic orders, artificial multiferroic heterostructures were introduced to the mix and are now playing a major role [5]. Here, the ferroelectric and magnetically ordered systems are coupled in multilayers, which is triggered via interface-based interactions, as depicted in figure 1 . The possibilities in the symmetry of the heterostructures, i.e. two dimensional based interface or involving nano-sized pillar self-assembled architectures have been reviewed in detailed previously [3], thus here we restrict ourselves to the most recent development in the design and integration of these multilayers into emerging technologies discussed in the following sections.

a Strain coupling

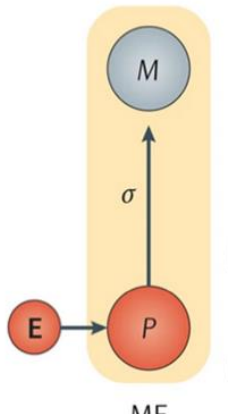

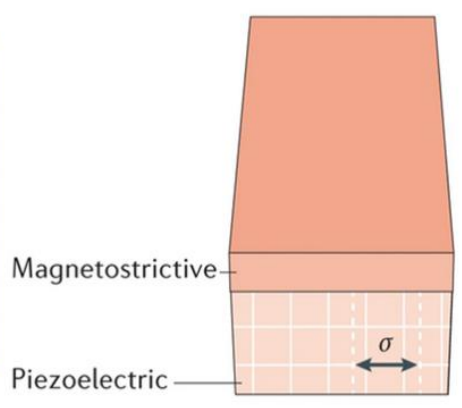

Piezoelectric b

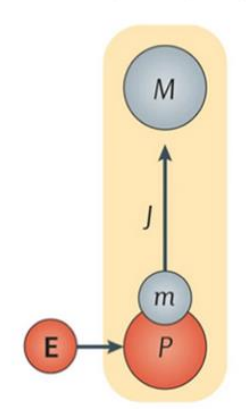

$\mathrm{MF}$

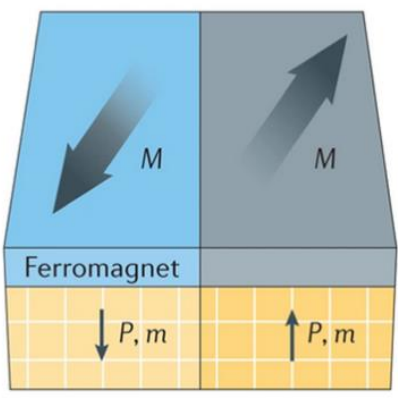

Ferroelectric antiferromagnetic 
FIGURE 1| Artificial magnetoelectric multiferroic heterostructures based strain coupling (a) and on interfacial magnetic exchange (b). Reprinted with permission from [1]. Copyright (C) 2016, Springer Nature.

\subsection{Energy efficient spintronics}

The demand for smaller and energy-efficient devices is driving the integration of complex oxides into new device architectures with reduced energy consumption. With the technological burst that followed the discovery of giant magnetoresistance [36,37], magnet-based data storage devices are at the forefront of current research. In state-of-the-art device concepts, spin currents replace heat- or magnetic field- assisted switching of the magnetic bit. Spin transfer torque (STT) technology is robust and offers ultrafast dynamics for various applications [38,39]. Spin currents directly generated via the spin Hall effect in heavy metals electrodes, so-called spin-orbit torques (SOT) are also emerging as technology relevant magnetic bit manipulation process [40-42]. Both STT- and SOT-based switching, however, dissipate significant energy through Joule heating, which, ultimately limits their integration into ever-smaller device architectures.

Ferroelectric-based memory devices offer the advantage of a non-volatile polarization state that can be controlled using a low-energy-consuming electric field. Electroresistance then allows the direct reading of the polarization state by monitoring the tunneling current across the ferroelectric $[43,44]$. Here however, interface related effects can drastically alter the polarization state in the ultrathin regime and hinder the implementation of ferroelectric materials in the required metal-ferroelectric-metal capacitor design. Uncompensated bound charges at ferroelectric epitaxial interfaces trigger a depolarizing field [45], which in return can suppress the ferroelectric behavior in the application relevant ultrathin films $[46,47]$.

The combined ferroelectric and magnetic states in artificial multiferroic heterostructures offer the possibility to control with an electric field a robust and easy to read magnetic state in the ultrathin regime. Here, we review the advances in the field of electrical control of magnetization in such architectures.

\section{Acting on magnetic order with electric fields in multiferroic heterostructures}

The development focus for the field of multiferroic heterostructures primarily lies in systems coupled through strain and interlayer magnetic exchange [3]. This is because the largest converse magnetoelectric coefficients and most reasonable switching times reported to date are observed in these 
systems $[48,49]$. Single phase multiferroics that are operable at room temperature, such as $\mathrm{BiFeO}_{3}$, tend to have low converse magnetoelectric coefficients $[1,50,51]$ that need to be enhanced to facilitate real applications.

\subsection{Electric field control of magnetic anisotropy}

In this section, we focus on the use of ferroelectric and piezoelectric crystals to modify or switch magnetocrystalline anisotropy in an adjacent magnetically ordered coupled layer, most commonly through the application of strain by the piezoelectric layer and taking advantage of magnetostriction in the ferromagnet. A magnetostrictive ferromagnetic layer is strained by a piezoelectric/ferroelectric crystal $[52,53]$ to produce changes in magnetic anisotropy [54-57] and control the associated energy landscape through magnetostriction, as represented in Figure 2a.

The magnetostriction coefficient, usually denoted as $\lambda_{i j k l}$, describes how a material transduces magnetization into strain according to the relation $\epsilon_{i j}=\lambda_{i j k l} \boldsymbol{m}_{k} \boldsymbol{m}_{l}=-C_{i j m n}^{-1} B_{m n k l} \boldsymbol{m}_{k} \boldsymbol{m}_{l}$, where $C_{i j m n}$ is the stiffness tensor, $B_{m n k l}$ is the magnetoelastic energy, and $\boldsymbol{m}_{k}, \boldsymbol{m}_{l}$ are the direction cosines of the magnetization $[58,59]$. Physically, this originates from changes in the band structure of the material due to the mechanical distortion. Effectively, as a crystal is strained and the atoms move, the band structure will change which can deplete/add to the spin-dependent density of states of a ferromagnet [60-62] . Macroscopically, this is observed as the change in magnetocrystalline anisotropy per change in strain, $B_{m n k l}=\frac{\partial \boldsymbol{K}_{m n}}{\partial \epsilon_{k l}}$, where $\boldsymbol{K}_{i j}$ is the magnetic anisotropy, and $\epsilon_{k l}$ is the strain [63]. As this is an effect of the magnetic anisotropy energy, it is proportional to the spin-orbit coupling of the material [62] and ferromagnets with large spin-orbit coupling, like rare earth containing materials, tend to also exhibit large magnetostriction coefficients. Additionally, materials that are mechanically isotropic (i.e. the ratio of components of $C_{i j m n}$ approach 1 or the shear modulus goes to 0 ) show larger magnetostriction values since $\lambda$ is proportional to $C^{-1} B$. Experiments with strain-mediated composite magnetoelectrics usually employ conductive magnets with large magnetostrictive coefficients $(\lambda)$ such as rare-earth containing Terfenol-D [52,64] $\left(\lambda \sim 1200\right.$ ppm), Galfenol $\left(\mathrm{Fe}_{1-x} \mathrm{Ga}_{x}\right)[65,66](\lambda \sim 250 \mathrm{ppm})$, FeRh [67] (ferromagnetantiferromagnet transition), CoFeB $[64,68](\lambda \sim 50 \mathrm{ppm})$, and Ni [69] $(\lambda \sim-34 \mathrm{ppm})$.

One static demonstration of this concept has been the imprinting of ferroelectric domains from a substrate onto a magnetostrictive film via strain-mediation (see section 3.2 for more on domain imprint). By depositing magnetostrictive CoFe on $\mathrm{BaTiO}_{3}$ substrate, the in-plane strain state of $\mathrm{a}_{1}-\mathrm{a}_{2}$ domains in the $\mathrm{BaTiO}_{3}$ create localized easy axes in the magnetostrictive magnet, effectively mapping the polarization 
of the substrate directly into a magnetic domain (Figure 2b) [70]. These imprinted magnetic domains have been seen to persist unambiguously through the temperature-driven phase transitions of $\mathrm{BaTiO}_{3}[71]$ and the effect has been noted with other magnetic films, such as $\mathrm{La}_{1-x} \mathrm{Sr}_{x} \mathrm{MnO}_{3}$ (LSMO) and $\mathrm{CoFe}_{2} \mathrm{O}_{4}$ [72]. Beyond this, magnetic domain imprinting has been used to control domain walls in the pre-switching regime, as the magnetic domain will move with the ferroelectric domain [73], and this phenomenon will be discussed further in section 3.

Magnetoelectric switching of magnetic anisotropy with the use of magnetostrictive ferromagnets has been able to achieve energy dissipations per area per switch on the order of $1-100 \mu \mathrm{Jm}^{-2}$ [69], making these devices competitive for post-Si memory and logic applications [74-76] . While much of the early work in strain-coupled multiferroics was done using bonded piezoelectric transducers $[77,78]$, scale devices necessitate thin film magnets deposited on high-strain piezoelectric crystals, typically relaxor-type $\mathrm{PbTiO}_{3}$ (PTO) derivatives such as $\mathrm{Pb}(\mathrm{Zr}, \mathrm{Ti}) \mathrm{O}_{3}$ (PZT) $[69,79,80]$ and $\mathrm{Pb}(\mathrm{MgNb}) \mathrm{O}_{3}-\mathrm{PbTiO}_{3}$ (PMN-PT) $[68,81,82]$. Strain mediation has widely been used to manipulate the energy landscape in composite materials [83], changing coercive fields and the depth of the potential well along the easy axis, (an example of which is shown in Figure $2 c$ ) however full switching of the anisotropy axis is much rarer $[49,84]$ and will be discussed further below.

Strain mediated magnetoelectric coupling is widely used for the production of extremely sensitive magnetic field detectors, particularly for biomedical applications [85-87]. In addition to the directional dependence of magnetization in strain-mediated composites, small changes in linear anisotropy energies can be detected via ferromagnetic resonance (FMR), shown in Figure 2d, and acoustic resonance of a cantilever structure $[85,87-89]$. These kind of multiferroic antennae have demonstrated frequency tunability in the $\mathrm{GHz}$ regime on the order of $\Delta f \cong 6 \mathrm{GHz}$ [88]. The use of this technology for biomagnetic sensors is extremely promising, with significant work in the field having progressed to devices testable in vivo $[90,91]$. 


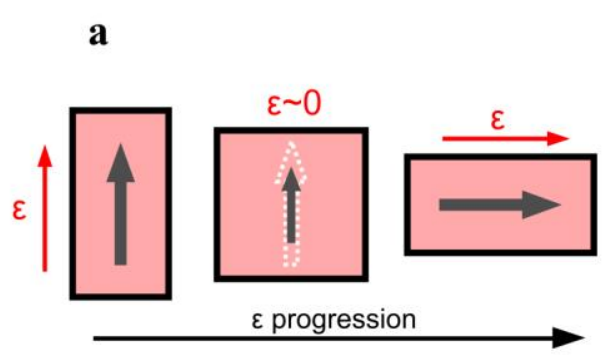

c

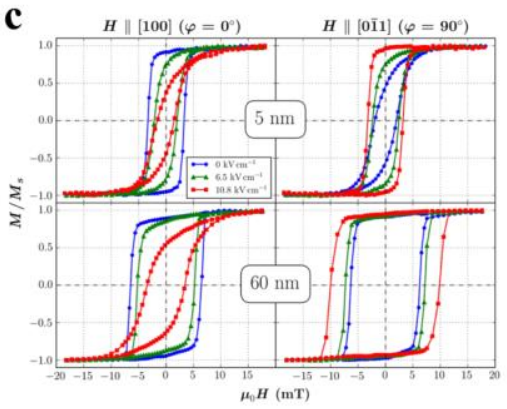

b

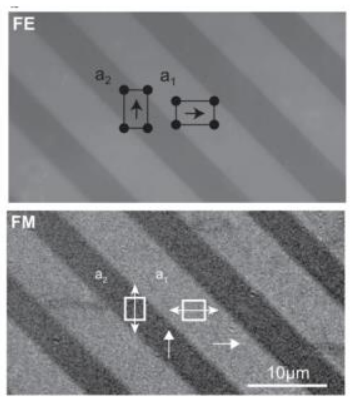

d

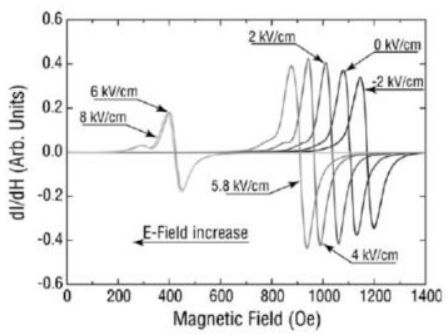

e
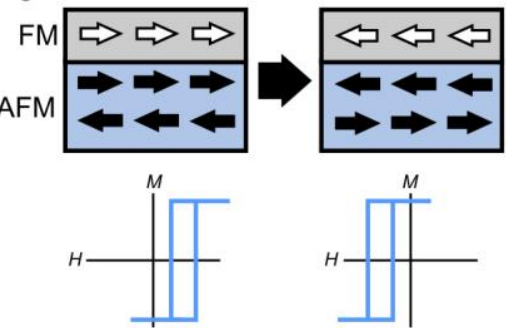

f

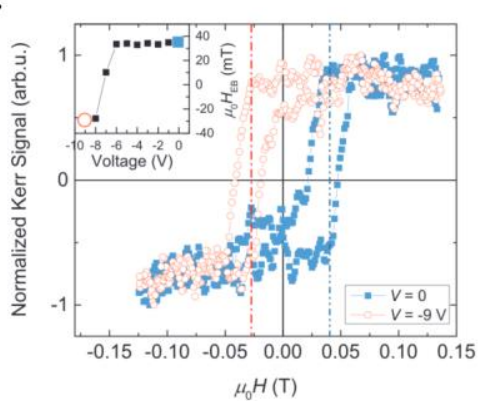

FIGURE 2| Strain- and exchange-coupled multiferroic composites. (a) Diagram showing how strain-coupled composites operate, where a strain is generated by a piezoelectric layer and transduced into a magnetization by the magnetostrictor. The figure shows both enhancement of uniaxial anisotropy due to strain and a potential switching pathway for a composite structure. (b) Birefringent (top) and magneto-optical Kerr effect (MOKE, bottom) images showing domain mapping from ferroelectric to ferromagnetic domains based on the in-plane strain state. Reprinted with permission from [54]. Copyright (C) 2012, Springer Nature. c) Hysteresis of a uniaxial Fe0.81Ga0.19/PMN-PT strain coupled composite, where the magnetic anisotropy is modulated by the application of an electric field. Reprinted with permission from [92]. Copyright 2020 by the American Physical Society. (d) Shift in FMR resonance as a function of applied electric field in FeGaB/PZN-PT. Reproduced with permission from [88] (copyright 2009 Wiley-VCH Verlag $\mathrm{GmbH} \&$ Co. KGaA). (e) Diagram showing a switching of the exchange bias field from an antiferromagnet, which is driven by the magnetoelectric effect in an antiferromagnetic exchange-coupled heterostructure. (f) MOKE hysteresis loop of exchange biased $(\mathrm{CoPd})_{n} / \mathrm{Cr}_{2} \mathrm{O}_{3}$, where the bias field is switched with a voltage and magnetic field pulse Reprinted with permission from [93]. Copyright @ 2010, Springer Nature.

\subsection{Electric field control of exchange bias}

Due to the mutually exclusive conditions for the onset of simultaneous ferromagnetic and ferroelectric order in a material (refer to section 1 and [8]), most single-phase multiferroic materials exhibit a ferroelectric order coexisting with an antiferromagnetic state. Therefore, single-phase multiferroic materials, such as $\mathrm{BiFeO}_{3}$, tend to have low converse magnetoelectric coefficients due to their small ferromagnetic moment that results from canted antiferromagnetic order [1,50,51,94,95]. Thus, conductive ferromagnetic layers, such as Co [96], CoFe [97,98] and $\mathrm{Ni}[69]$ are employed and exchange 
coupled to the multiferroic magnetic order. This effectively amplifies the weak magnetic moment of the multiferroic. In so-called exchange coupled multiferroic heterostructures, a single-phase magnetoelectric antiferromagnetic multiferroic is coupled to a ferromagnetic layer through interfacial coupling. The exchange bias, see Figure 2e, is a phenomenon that occurs when a thin ferromagnet is in contact with the surface of an antiferromagnetic crystal. Below the ordering temperature of the antiferromagnetic state, the Néel temperature, the relatively hard spins of the antiferromagnetic surface interact with the soft spins in the ferromagnet, pinning the magnetization direction to that of the surface spins in the antiferromagnetic lattice [99]. This results in a significant broadening of the ferromagnetic hysteresis under magnetic field application, an enhancement in the coercive field, and a shift of the loop along the field direction called exchange bias field. At zero magnetic field, the ferromagnet is robustly pinned into one state by the bias field. Here, a magnetoelectric switching event can trigger a change in the antiferromagnetic state and lead to a net exchange coupled magnetization switch. The efficiency of this switching mechanism has been demonstrated in heterostructures based on prototypical antiferromagnetic magnetoelectric materials $\mathrm{Cr}_{2} \mathrm{O}_{3}[100,101]$ and $\mathrm{BiFeO}_{3}[97,102-104]$. An example of this switching using $\mathrm{Cr}_{2} \mathrm{O}_{3}$ is shown in Figure $2 \mathrm{f}$.

$\mathrm{Cr}_{2} \mathrm{O}_{3}$ is a non-ferroelectric antiferromagnetic material which shows a linear magnetoelectric effect above room temperature $[9,10]$. It is a uniaxial antiferromagnet pointing along the c-axis of the hexagonal unit cell, and the magnetic cations are arranged in such a way that a c-plane terminated crystal can be engineered to produce a single, global, perpendicular surface magnetization [105] . This out-ofplane antiferromagnet would exchange couple to a ferromagnetic film or multilayer with perpendicular magnetic anisotropy, in contrast to most in-plane AFM/FM systems, creating an out-of-plane magnetized multiferroic heterostructure. Electric-field-driven switching of this configuration was first demonstrated in bulk crystals with out-of-plane ferromagnet $(\mathrm{Co} / \mathrm{Pt})_{\mathrm{n}}$, however it required a static magnetic field to achieve full switching of the surface magnetization $[101,105]$. Linear magnetoelectric switching is defined by a critical energy product of electric field and magnetic field [10], meaning magnetoelectric switching of $\mathrm{Cr}_{2} \mathrm{O}_{3}$ requires either substantial static magnetic fields $[106,107]$ or extraordinarily large voltages. This presents a significant engineering challenge for scaling of devices, as a $\mathrm{Cr}_{2} \mathrm{O}_{3}$ thin film must be able to withstand voltages large enough for switching, and significant work is currently focused on increasing the breakdown voltage or decreasing the switching threshold for thin films $[108,109]$. Thus far, magnetoelectric switching in thin film heterostructures has required substantial magnetic fields on the order of 0.6-1 T $[93,106,107]$, but the technology remains promising for scale multiferroic devices. 
In room temperature ferroelectric antiferromagnetic $\mathrm{BiFeO}_{3}$, the antiferromagnetic structure can cant and result in a weak moment that forms a long-range spin cycloid [110]. With enough epitaxial strain, a uniform canted moment emerges [111]. The antiferromagnetic and canted moment structure have been observed to track the ferroelectric polarization, thus, the moments of an exchange coupled ferromagnet not only amplify the effective moment but also correlate to the ferroelectric domain structure of the multiferroic $[21,23,96,98,102,112,113]$. This coupling between the magnetic and ferroelectric domain structure then permits the electric field control of the magnetization through the control of the ferroelectric polarization. This has been leveraged in $\mathrm{BiFeO}_{3}-\mathrm{LSMO}$ heterostructures and the sign of the bias field could be reversed after electrical switching $[103,104]$. In this system, however, the exchange bias field is not large enough to fully switch the magnetization of the ferromagnet, requiring the use of other magnetic layers to realize deterministic switching.

\subsection{Electric field induced magnetization reversal}

In strain-coupled multiferroics, the use of epitaxy to maximize strain transfer to the magnetostrictive layer has enabled electric field-controlled, non-volatile $90^{\circ}$ switching of the ferromagnetic magnetization in a Galfenol (FeGa) film [65] (Figure 3a,b). This, however, may be the limit for purely strain-based devices as symmetry considerations state only $90^{\circ}$ switching should be possible with a single voltage pulse. Experimental reports have demonstrated that enough magnetoelastic energy can be generated to drive $90^{\circ}$ reorientation of anisotropy $[54,65,82]$, but $180^{\circ}$ switching, which is desired for maximum readout, may not be achievable unless sequential voltages are applied $[114,115]$. As an engineering challenge, this problem can be circumvented if material symmetries are broken by external stimuli or clever architectures utilizing the switching kinetics of the ferroelectric [114]. One way this can be accomplished is through external magnetic fields [116-118] or hybrid magnetoelectric-spintronic devices $[119,120]$, which are discussed in section 4. 

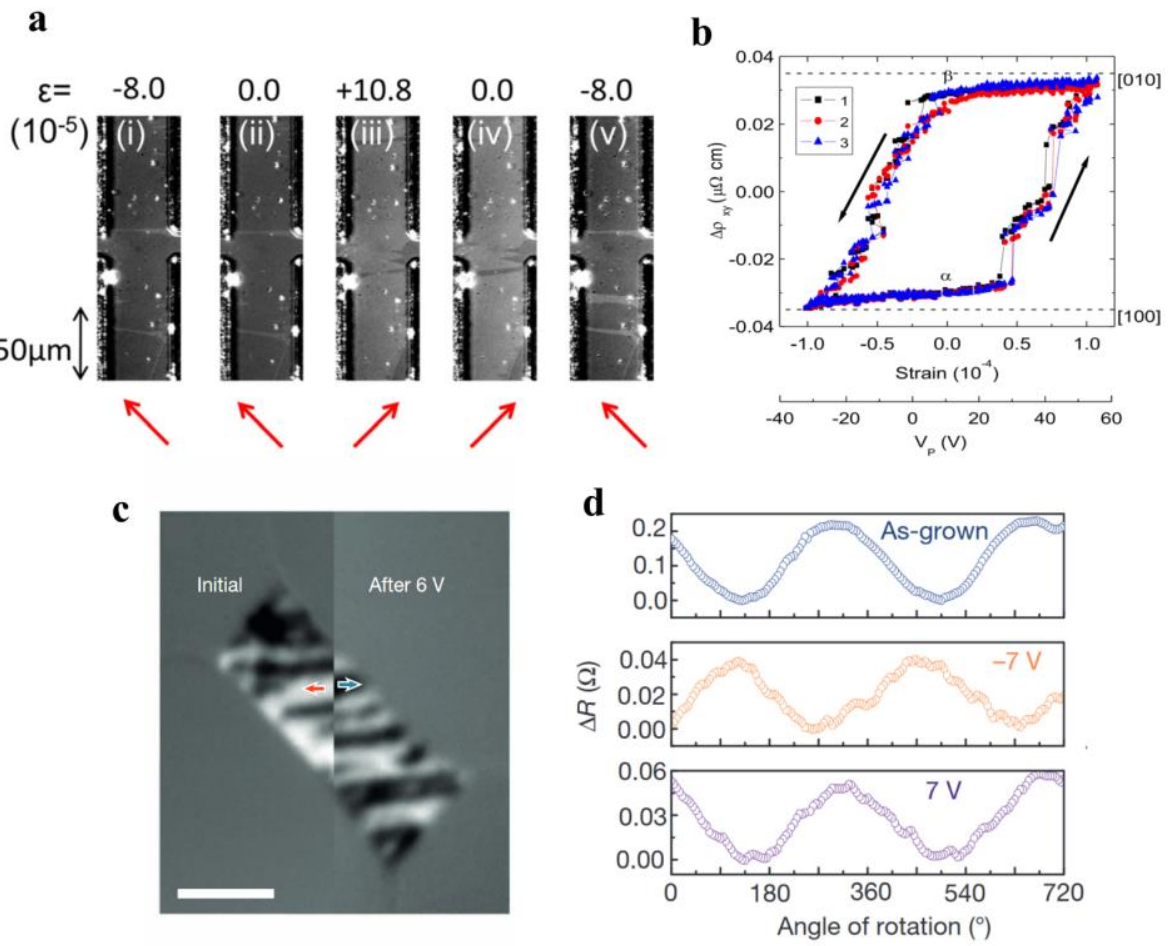

FIGURE 3|Magnetoelectric switching of magnetization. a) Magneto-optical Kerr effect (MOKE) micrographs of a strain-coupled composite multiferroic consisting of magnetostrictive $\mathrm{Fe}_{0.81 \mathrm{Ga}} \mathrm{G}_{0.19}$ epitaxially grown on a GaAs substrate and bonded to a piezoelectric transducer. We see that, as the device is cycled through a full ferroelectric loop, the magnetization is deterministically switched between the two strain states. b) Transverse anisotropic magnetoresistance (AMR) showing repeatable resistive switching of the device. Reprinted with permission form [65]. Copyright 2012, AIP Publishing LLC. c) X-ray magnetic circular dichroism photoemission electron microscopy (XMCDPEEM) images of a CoFe/ $\mathrm{BiFeO}_{3}$ exchange-coupled multiferroic showing deterministic switching of the magnetization that follows the ferroelectric domain pattern of the $\mathrm{BiFeO}_{3}$. d) Low-field AMR versus applied voltage, showing $180^{\circ}$ switching of magnetization under volatage. The upper, middle and lower panels show AMR from the as-grown (asdeposited) state, after -7 V and after +7 V. Reprinted with permission from [97]. Copyright $@$ 2014, Springer Nature.

Full switching of magnetization induced by an electric field has been observed in exchange coupled $\mathrm{BiFeO}_{3}$-ferromagnetic heterostructures, shown in Figure 3c,d. The magnetization of the ferromagnetic layer is strongly coupled to the antiferromagnetic axis, whose orientation depends on the ferroelectric polarization direction. Deterministic switching has been observed and studied primarily using CoFe metallic ferromagnets [97,102,121,122] using both microscopy [97] and transport measurements $[102,122]$. This deterministic switching event which requires no external fields has spurred the development of multiferroic computing technologies based on the material system $[75,76]$, as well as 
significant efforts to increase its efficiency $[122,123]$. Its implementation into energy efficient device heterostructures will be described in section 4 .

\section{Ferroic domains and domain imprint in thin film heterostructures}

In multiferroic heterostructures, energy efficient functionalities rely on the ability to alter the ferroelectric domain architecture using an electric field in a controlled fashion once integrated in the ferroelectric/ferromagnetic multilayer. Ferroelectric domain architectures define the switching mechanism, they can also be transferred into an adjacent ferromagnetic layer and therefore drive the magnetoelectric behavior of the multiferroic multilayer. Engineering of domains and corresponding domain walls is the key towards reliable magnetoelectric switching events.

\subsection{Ferroelectric domain engineering}

Epitaxy - Because of the lattice-polarization coupling in ferroelectrics, the ferroelectric domain configuration in epitaxial thin films is dictated by the geometry and epitaxial strain, imposed by the selected single-crystal substrate. Substrate step edges are often preferential nucleation sites for ferroelectric domains [124], therefore substrate orientation and miscut angle become tuning parameters to control the degeneracy of domains $[125,126]$. In-plane strain anisotropy induced by the substrate often results in anisotropic stripe domain patterns $[127,128]$. Epitaxial strain, in extreme cases, can even induce polarity in otherwise non-polar structures, such as $\mathrm{SrTiO}_{3}$ [129], EuTiO 3 [130], $\mathrm{SrMnO}_{3}\left[131,132\right.$ ] or $\mathrm{BaSnO}_{3}$, $\mathrm{SrSnO}_{3}$ [133]. Domain engineering in ferroelectric thin films using epitaxial strain has been reviewed in great detail, see [45] for references. Here, we highlight some recent development involving electrostatic boundary conditions and surface symmetry and atomic terminations to achieve a predefined polarization state.

In the particular case of multiferroic magnetoelectric $\mathrm{BiFeO}_{3}$ thin films, the configuration of ferroelectric domains can be fully controlled by the choice of the crystallographic orientation of the substrate: thin films have one, two or four allowed polarization variants when grown on (111), (110) and (001) orientations of the lattice matching $\mathrm{SrTiO}_{3}$ substrate, respectively [125]. High interfacial strain gradient can produce mosaic ferroelectric domains in $\mathrm{BiFeO}_{3}$ that can be turned into stripes upon substrate annealing [134]. It is worth noting that the antiferromagnetic order in multiferroic $\mathrm{BiFeO}_{3}$ is strongly 
correlated with the ferroelectric domain state and also depends on epitaxial strain: spin cycloid can be suppressed by epitaxial strain $[135,136]$. $\mathrm{BiFeO}_{3}$ epitaxial films thus provide a unique platform towards the antiferromagnetic order parameter engineering using epitaxial strain in thin films. Its antiferromagneticaxis orientation can be tuned [137] from in-plane to out-of-plane by compressive and tensile strain, respectively. Furthermore, once stabilized in a single ferroelectric and magnetic domain state, the magnetic polarity in $\mathrm{BiFeO}_{3}$ domains is inverted upon $180^{\circ}$ ferroelectric switching on the macroscopic scale $[138,139]$.

Electrostatics - Depolarizing field emerges with the accumulation of the ferroelectric bound charges at the surfaces of ferroelectric thin films. Oppositely oriented to the polarization direction, it usually has a detrimental impact on the polar properties of the films in the technology-relevant ultrathin regime. The depolarizing field can cause the polarization suppression $[47,140,141]$ and limit the integration of ultrathin ferroelectric materials into devices and is therefore often viewed in a negative light. As most ferroelectric systems grow epitaxially in the ferroelectric phase [141,142], the depolarizing field already impacts the domain state during the design of functional heterostructures based on ferroelectric films [46]. Nevertheless, the progress in epitaxial design and nanoscale ferroelectric domain probe capacity recently allowed the integration of depolarizing field effects into the domain engineering process to tailor ferroelectric domains in a controlled manner with desired electrostatic boundary conditions $[143,144]$.

In addition to the impact of the bound charge screening on the ferroelectric polarization state in the ultrathin regime, the electrode material selection and its corresponding work function drastically impact the ferroelectric bulk properties. Any asymmetry in the electronic and chemical environments of a ferroelectric layer results in an internal built in field $[145,146]$, which affects the polarization orientation and induces a preferred polarization direction and retention problems $[147,148]$. The insertion of a dielectric material within the ferroelectric capacitor architecture results in reduced bound charge screening efficiency and an onset of the depolarizing field, which induces a domain nucleation and leads to a multi-domain state in ferroelectric layer. It attenuates the built-in field influence on the ferroelectric hysteresis loop. Thickness control of the dielectric layer allows for tuning of the depolarizing field strength and the corresponding domain formation [144]. Furthermore, the existence of domains in an as-grown state and diminished dielectric leakage further improves the switching performance $[143,144,147]$. Such an approach previously restricted to prototypical tetragonal ferroelectric $\left(\mathrm{BaTiO}_{3}, \mathrm{PZT}\right.$ and PTO) has been used to engineer multiferroic domains in $\mathrm{BiFeO}_{3}$ films. Chen and co-workers [149] showed that ultrathin dielectric layer of $\mathrm{La}$-doped $\mathrm{BiFeO}_{3}$ radically alters domain state in $\mathrm{BiFeO}_{3}$. On $\mathrm{DyScO}_{3}$ substrates, when a 
conducting buffer is used, a uniform out-of-plane polarization sate is stable and the $\mathrm{BiFeO}_{3}$ systems exhibits $71^{\circ}$ stripe domain architecture. However, the insertion of the dielectric buffer results in the conversion towards a fully $109^{\circ}$-type stripe domain pattern, without any net out-of-plane polarization component and technology-relevant conducting $109^{\circ}$ domain walls $[150,151]$ (See Figure 4a-d).

Atomic surface termination and interface chemistry, symmetry - In the ultrathin regime, the polarization direction (up or down in a single domain state) and overall domain architecture can also be controlled by selecting an appropriate atomic surface termination of the substrate or buffer layer. Using chemical surface treatments or thermal annealing, the substrate atomic termination can be engineering. Considering perovskite $\mathrm{ABO}_{3}$ model system, the thin film (001) oriented growth can take place from either the $\mathrm{AO}$ of $\mathrm{BO}_{2}$ plane. In the case of $\mathrm{ABO}_{3}$ type ferroelectrics containing charged planes, there is an electrostatic potential step across the interface with the buffer. This results in a deterministic control on the direction of polarization of the film. For instance, because the $\mathrm{BiFeO}_{3}$ unit cell consists of charged planes, i.e. $(\mathrm{BiO})^{+}$and $\left(\mathrm{FeO}_{2}\right)^{-}$, the polarization can be predefined by selecting the buffer surface termination for the growth. On negatively charged $\left(\mathrm{MnO}_{2}\right)^{-0.7}$ planes of LSMO buffer, the growth starts with positively charged $(\mathrm{BiO})^{+}$planes. The resulting potential sets the polarization in the upwards direction. The deposition on $\left(\mathrm{La}_{0.7} \mathrm{Sr}_{0.3} \mathrm{O}\right)^{+0.7}$ plane results in downward oriented films [152]. Because the stacking sequence in atomic plane can propagate during the growth of several successive perovskite materials, the design of multilayer with predefined polarization configuration [141], i.e. with parallel or antiparallel relative polarization state, is possible as shown in Figure 4e-g. Moreover, different interfacial atomic sequences and corresponding electrostatic potential, also results in a modulated magnetoelectric effect and exchange bias in oxide multilayers [153]. The atomic surface termination and symmetry provide handle on the polarization state from the bottom interface during the multilayer design process itself, adding a degree of freedom for the design of domain architecture and the surface topography of the film [154].

In order to stabilize ferroelectricity in the ultrathin regime, polar metals crystallizing in noncentrosymmetric point groups compatible with ferroelectric symmetries are emerging as a promising alternative to standard metals. Their intrinsically broken parity can even help to surpass critical thickness [155]. They have been proposed as key elements towards the suppression of the critical thickness in ferroelectrics once integrated in technology-relevant capacitors. The scarcity of polar metals, that are structurally compatible with application-relevant ferroelectrics, however, limits their integration into ferroelectric base devices. Alternatively, the chemistry at the top interface of the ferroelectric material can be tuned in order to achieve a deterministic polarization state [156]. The importance of tunable 
electrostatics at ferroelectric $\mathrm{BiFeO}_{3}$ films surfaces was explored in a reversible aqueous switching. Depending on the $\mathrm{H}^{+} / \mathrm{OH}^{-}$concentration of the solution in direct contact with the film, the formation of polarization-selective chemical bonds at the interface is controlled, as shown in Figure 4h-i.

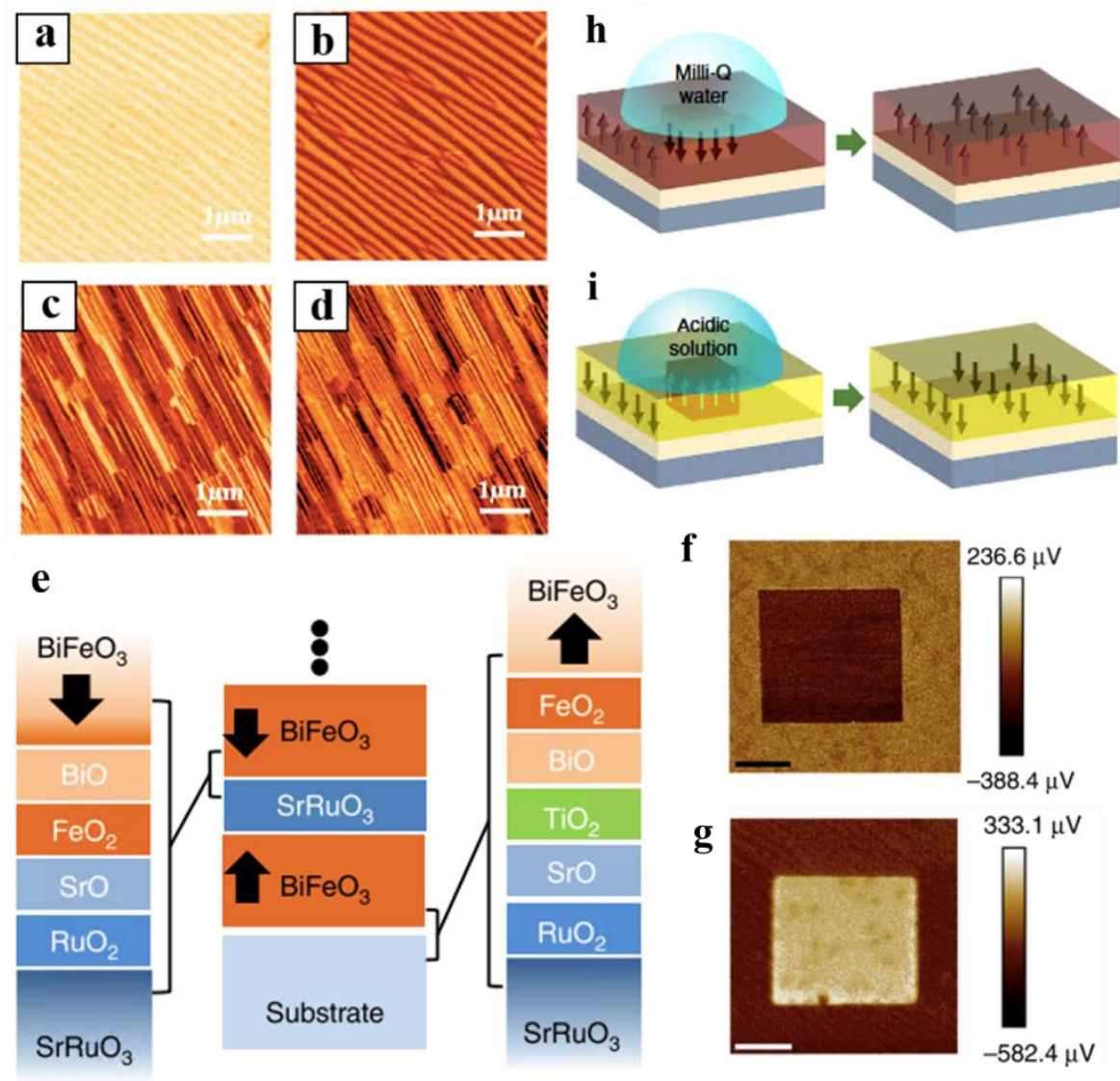

FIGURE 4| (a-d) $\mathrm{BiFeO}_{3}$ domain engineering by insertion of dielectric La-doped $\mathrm{BiFeO}_{3}$ buffer layer. Out-of-plane (a) and in-plane (b) Piezoresponse force microscopy (PFM) images of $\mathrm{BiFeO}_{3} 71^{\circ}$ stripe domain structure in $\mathrm{BiFeO}_{3} / \mathrm{SRO} / \mathrm{DSO} .109^{\circ}$ stripe domains appear upon the insertion of La-doped $\mathrm{BiFeO}_{3}$ in vertical (c) and lateral (d) PFM images. Reprinted with permission from. [149]. Copyright (C) 2017, American Chemical Society. (e-g) Engineering ferroelectric multilayers with user-defined up/down polarization sequence (e): A-site growth and upwards polarization of $\mathrm{BiFeO}_{3}$ is achieved by insertion of a $\mathrm{TiO}_{2}$ monolayer. B-site growth and downwards polarization of $\mathrm{BiFeO}_{3}$ is achieved by $\mathrm{SrO}$ self-termination of $\mathrm{SRO}$ buffer, confirmed by PFM scans (f) and (g), respectively. Reprinted with permission from [141]. Copyright (c) 2017, Springer Nature (h-i) Water poling of ultrathin ferroelectric $\mathrm{BiFeO}_{3}$ : polarization is reversed to the upward ( $h$ ) and downward (i) after exposing the film to water and acidic solution, respectively. Reprinted with permission from [156]. Copyright @ 2018, Springer Nature.

\section{2 Domain imprint}


The interface between ferroelectric and ferromagnetic thin films can enable magnetoelectric coupling. In particular, ferroelectric domains can be imprinted into the magnetic state in an adjacent layer. There are three distinct ways to achieve artificial magnetoelectric coupling [5]: via (i) strain, (ii) direct (spin) exchange, and (iii) charge coupling, see section 2. In strain coupled artificial multiferroics, piezoelectric crystal or thin film and magnetostrictive layer are elastically coupled leading to controllable magnetoelastic anisotropy due to propagation of electrostrain [70,157-164] with a key requirement of strong elastic pinning of magnetic domain walls onto ferroelectric domain walls [165]. In exchange-biased multiferroic composites, the interaction occurs between a ferromagnet and intrinsic multiferroic with uncompensated antiferromagnetic order, such as $\mathrm{BiFeO}_{3}$ [21,98,166,167], $\mathrm{YMnO}_{3}$ [168] and $\mathrm{LuMnO}_{3}$ [118]. The electric field changes both the polarization and the direction of antiferromagnetic spin ordering in a multiferroic. This allows a direct exchange effect between antiferromagnetic and ferromagnetic spin ordering. Although the physics of exchange coupling is different from strain coupling, both produce lateral modulations of magnetic anisotropy leading to domain transfer. Note that because strain is uniaxial, the electrical directional control of a magnetic state, i.e. $180^{\circ}$ electric-field-induced magnetization reversal, has been restricted to exchange-coupled systems. Charge-coupled artificial multiferroics make use of the ferroelectric field effect: non-volatile bound charges at the ferroelectric interface are screened by the ferromagnetic layer, leading to either accumulation (hole doped) or depletion (electron doped) states in ferromagnet when the polarization is pointing away or towards the ferromagnet, respectively. If the ferromagnet has multiple accessible ground states, this might result in drastic alterations of magnetization and domain imprint $[79,169-175]$. This mechanism is different from the two mentioned previously, because changes in magnetization are limited to interfaces, up to a screening length $[175,176]$. However, in the ultrathin regime (especially when superlattices are considered), full domain transfer could be achieved.

Most recent studies on domain imprint have focused on the dynamics investigation aiming to understand the origin of the domain imprint and the domain pattern evolution upon the application of electric field. De Luca and coworkers [177] investigated magnetoelectric coupling dynamics between exchange-coupled $\mathrm{BiFeO}_{3}$ and ferromagnetic CoFe using optical SHG and magnetic force microscopy (MFM) techniques operando; see Figure 5a-f . An electric field in the order of coercive field of $\mathrm{BiFeO}_{3}$ is required to activate the domain pattern transfer. In another work, magneto-optical Kerr effect (MOKE) microscopy was employed to show that ferroelectric and ferromagnetic domain walls move in unison upon the application of out-of-plane electric field pulses $[73,178,179]$. These experiments confirmed that the process is reversible and neither magnetic field, nor current are required. The domain wall velocity is hence 
fully determined by electric field strength and can reach several $100 \mathrm{~m} \mathrm{~s}^{-1}$ as predicted by numerical simulations [180]. Recent studies on strain-mediated magnetoelectric coupling revealed that rotations of the local magnetization upon electric field pulses are normally smaller than expected $90^{\circ}$ due to electrically driven shear strain [181], see Figure 5g-I. This guarantees a deterministic return to the initial magnetization direction with no possibility of magnetization reversal and offers the prospect of writing data both magnetically and electrically [181].

A novel route towards exact matching between ferroelectric and ferromagnetic domain patterns was proposed by Leo et al. [182] in bulk material. Complete magnetoelectric domain inversion was observed in multiferroic $\mathrm{Mn}_{2} \mathrm{GeO}_{4}$ and magnetoelectric $\mathrm{Co}_{3} \mathrm{TeO}_{6}$ : the ferroic order parameter in these materials is fully reversed in in each domain, but the initial domain pattern is perfectly reproduced. Figure $5 \mathrm{~m}$ shows how ferroelectric domains in $\mathrm{Mn}_{2} \mathrm{GeO}_{4}$ are inverted upon application of magnetic field. The observation is rationalized by the coupling between a complex set of order parameters where one of them is independently switched with an external field, while another retains the memory of the domain pattern. A related effect was observed in $\mathrm{Dy}_{0.7} \mathrm{~Tb}_{0.3} \mathrm{FeO}_{3}$ [183]: here multiferroic domains defined by multiple order parameters can be reversibly converted to multiferroic domain walls and back at a specified location within a non-multiferroic matrix. Such interconversion can be a key to tailoring elusive domain architectures in antiferromagnets. Although both effects have only been demonstrated at cryogenic temperatures, other materials exhibiting similar properties are anticipated to be discovered, owing to the generality of the two effects.

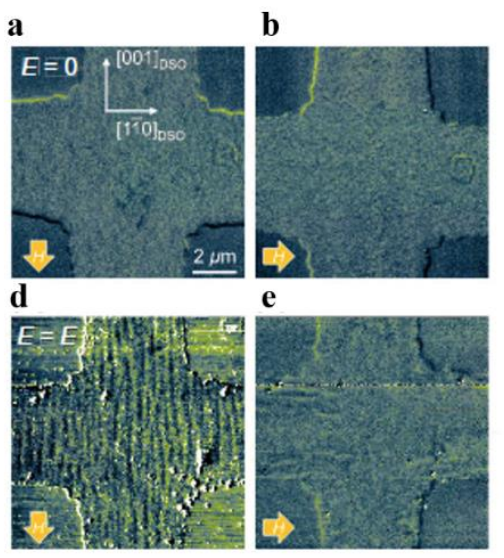

C
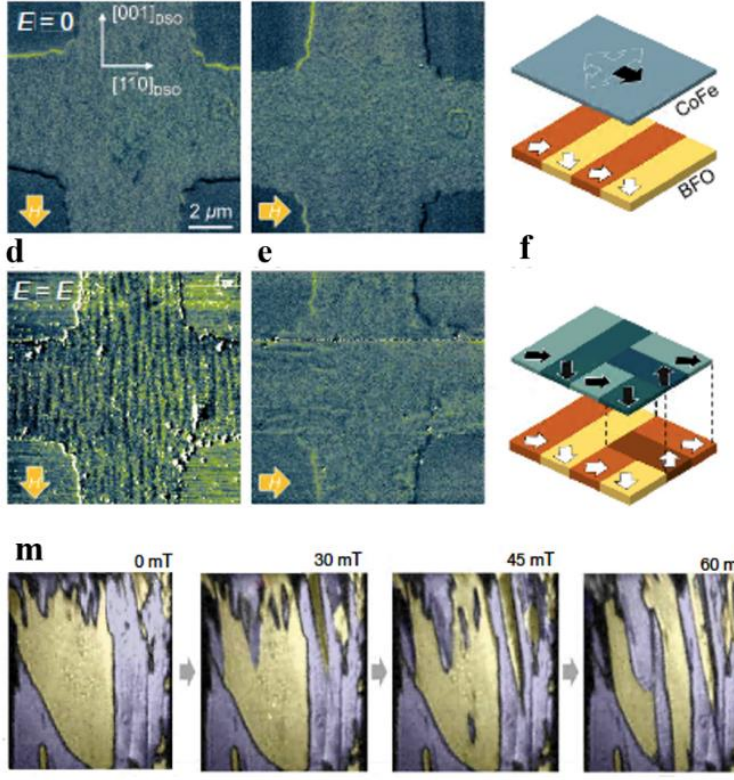

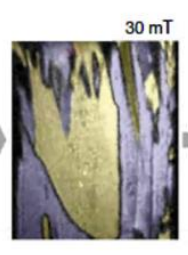

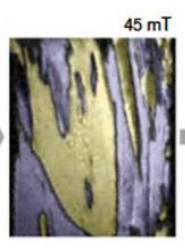

g
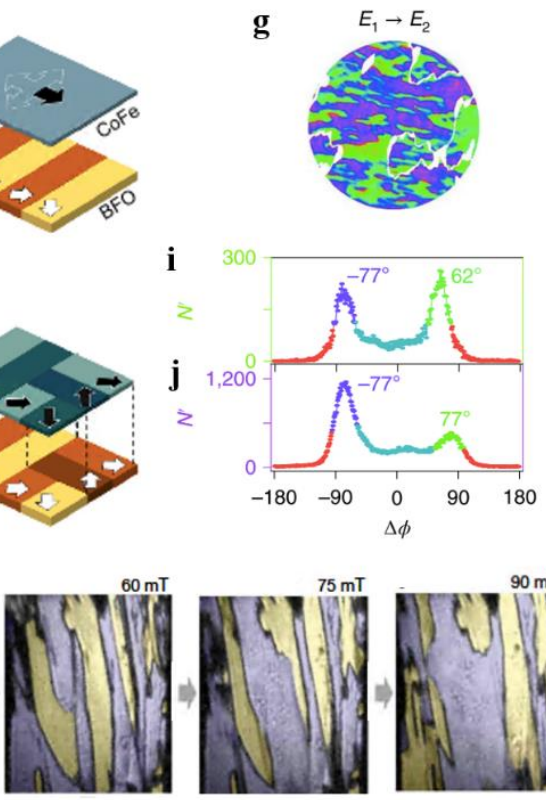

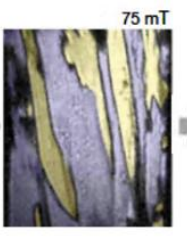

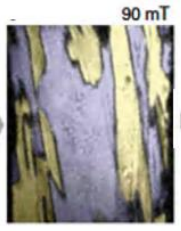
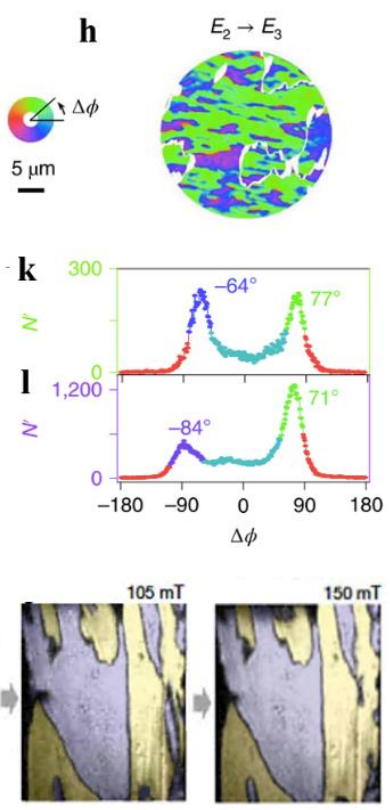
FIGURE 5| (a-f) Domain transfer from $\mathrm{BiFeO}_{3}$ to $\mathrm{CoFe}$. The spatially resolved MFM scans show ferromagnetic domains in CoFe with the magnetic field applied along $[001]_{D s o}(a, d)$ and $[1-10]_{D S O}(b, c)$. The pristine CoFe film yields a uniform in-plane magnetization indicating the suppression of the magnetoelectric $\mathrm{BiFeO}_{3} / \mathrm{CoFe}$ coupling as shown in (c). After application of an electric field $E \approx E c$ domain pattern transfer is activated (f). Reprinted with permission from [177]. Copyright 2018 by the American Physical Society. (g-I) Electrically driven shear strain effects in magnetoelectric switching of PMN-Pt/Ni heterostructure. 50- $\mathrm{mm}$-diameter maps $(\mathrm{g}, \mathrm{h})$ showing changes of magnetization direction $-180^{\circ} \leq \Delta \varphi \leq 180^{\circ}$ of each pixel upon electric field steps $(\mathrm{E} 1 \rightarrow \mathrm{E} 2)$ and $(\mathrm{E} 2 \rightarrow \mathrm{E} 3)$ anticipated to interconvert the hard and easy magnetization directions in Ni. Due to shear strain magnetization rotates by angles smaller than hitherto expected $90^{\circ}$, as shown by the number of pixels $N^{\prime}$ that undergo a change of magnetization direction $\Delta \varphi$, for pixels that are green $(\mathrm{i}, \mathrm{k})$ and purple $(\mathrm{j}, \mathrm{l})$ with modal angles specified. The data color represents a modal angle on the color wheel. Reprinted with permission form [181]. Copyright (C) 2019, Springer Nature. (m) Inversion of the ferroelectric domain pattern in multiferroic $\mathrm{Mn}_{2} \mathrm{GeO}_{4}$ evidenced by sequentially taken SHG images of out-of-plane $P_{z}$ domains at the given magnetic fields $\mathrm{Hz}$. Reprinted with permission from [182]. Copyright (C) 2018, Springer Nature.

\subsection{Domain wall engineering}

Domain architecture is also characterized by its domain walls configuration. Domain walls, separating different region of uniformly oriented order parameter, here ferroelectricity and ferromagnetism, can play an important role in functionalities of thin film heterostructures. The physical properties emerging at ferroelectric domain walls and the corresponding applications have been the subject of several reviews, refer to [184-186]. Here, we place the emphasis on the magnetoelectric systems and applications.

Tremendous progress has been achieved in the field of ferroelectric domain walls over the last couple of years. Ways of injecting ferroelectric domain walls [187-189] and controlling their motion $[188,190,191]$ as well as their speed [192] are now established. Sharma and coworkers [189] have demonstrated a prototype domain wall switch in $\mathrm{BiFeO}_{3}$ with three distinct states: no domain wall, neutral domain wall and charged domain wall as shown in Figure 6a-f. Domain wall detection is now possible not only via conventional scanning probe techniques [185], but also with optical SHG [193,194], Raman spectroscopy [195] as well as synchrotron X-ray diffraction [196]. Moreover, PZT domain walls were recently identified as optically active objects when interfaced with $\mathrm{MoS}_{2}$ flakes offering novel tunability of interfacial optical response [197]. All these advances point towards the future development of magnetoelectric devices based on domain walls.

Magnetic domain walls in ferroelectric matrix - Magnetoelectric coupling in heterostructures can alternatively be achieved through the domain walls. The importance of the domain wall role first emerged 
upon nanoscale investigation of $\mathrm{BiFeO}_{3}$ interfaced with $\mathrm{CoFe}$, which showed that exchange-bias directly depends on the type and crystallography of the domain walls in $\mathrm{BiFeO}_{3}$ [112]. An enhancement of the coercive field is observed regardless of the domain wall type, while exchange bias itself, manifesting by shifted magnetization hysteresis, is ascribed to uncompensated spins at $109^{\circ}$ domain walls exclusively [112], as seen in Figure 6g-l. The domain wall dynamics itself influences the domain imprint or domain coupling in artificial multiferroic heterostructures. The application of an electric field can induce a ferroelectric domain wall motion, releasing the imprint or magnetoelectric coupling locally until the domain coupling is recovered at a new ferroelectric domain wall location. This was used to control the magnetic exchange bias and magnetotransport properties in the model system $\mathrm{YMnO}_{3} / \mathrm{Py}$ [168] and $\mathrm{LuMnO}_{3} / \mathrm{Py}$ bilayers. Antiferromagnetic and ferroelectric domain walls in multiferroic manganites can be unclamped and change the sign of exchange bias after each successive electric pulse [118]. Such clamped domain walls, possessing multiple ferroic order parameters, can in turn be referred to as multiferroic domain walls [1]. They sometimes are the sole reason for magnetoelectric domain coupling, e.g. as in hexagonal manganites $\mathrm{h}-\mathrm{RMnO}_{3}$ [12]. Multiferroic domain walls have also been reported in multiferroics such as $\mathrm{MnWO}_{4}[198,199], \mathrm{RFeO}_{3}[200,201]$ or $\mathrm{TbMnO}_{3}$ [202]. A finite magnetic moment at the domain wall can sometimes accompany polar and antiferromagnetic domains as measured in h-ErMnO${ }_{3}$ [203], oxygen deficient $\mathrm{PbTiO}_{3}$ [204] or ferroelastic $\mathrm{TbMnO}_{3}$ [205]. We note that polar domain walls in nonferroelectric structures, e.g. Néel-like domain walls in iron garnet films [206], ferroelastic domain boundaries [207-209] have also been reported. All of this holds potential for magnetoelectric coupling in heterostructures right at the wall location in a non-magnetoelectric host compound.

Charged-domain-wall in vicinity of magnetic state - Bound charge accumulation at so called charged ferroelectric domain walls $[15,150]$ or ferroelastic boundaries $[210]$ creates another pathway to a magnetoelectric effects at domain walls: localized charge coupling can be induced at interfaces of metallic ferromagnets [170]. This could trigger electrically tunable localized interfacial reconstructions, leading to the ferromagnetic to antiferromagnetic phase transition in colossal magnetoresistive ferromagnets such as LSMO $[171,211]$. A challenge remains in the stabilization of charged domain walls and their unfavorable electrostatic configuration. The family of improper ferroelectric, i.e. geometric ferroelectrics $h$ - $\mathrm{RMnO}_{3}$ [15] or electronically-driven improper ferroelectric $\mathrm{CsNbW}_{2} \mathrm{O}_{9}$ [212] are prominent candidates for the design of functional and stable charged domain walls.

In proper ferroelectric systems, fully in-plane polarized systems may open new avenues towards stable charged domain wall design. In in-plane polarized system, the depolarizing field influence on the domain pattern does not depend on the layer thickness and may be linked to the domain width [30,31]. 
Stable periodic arrays of nominally charged domain walls have been recently reported in ultrathin Aurivillius films (see Figure 6m-0) [30], putting this class of layered ferroelectrics $[29,213]$ in the forefront of domain wall engineering research. These compounds can further host magnetic ions driving multiferroicity $[34,214-216]$. However, the impact of layered anisotropic structure $[217,218]$ and B-site doping on the domain wall electronic configuration remains to be explored.
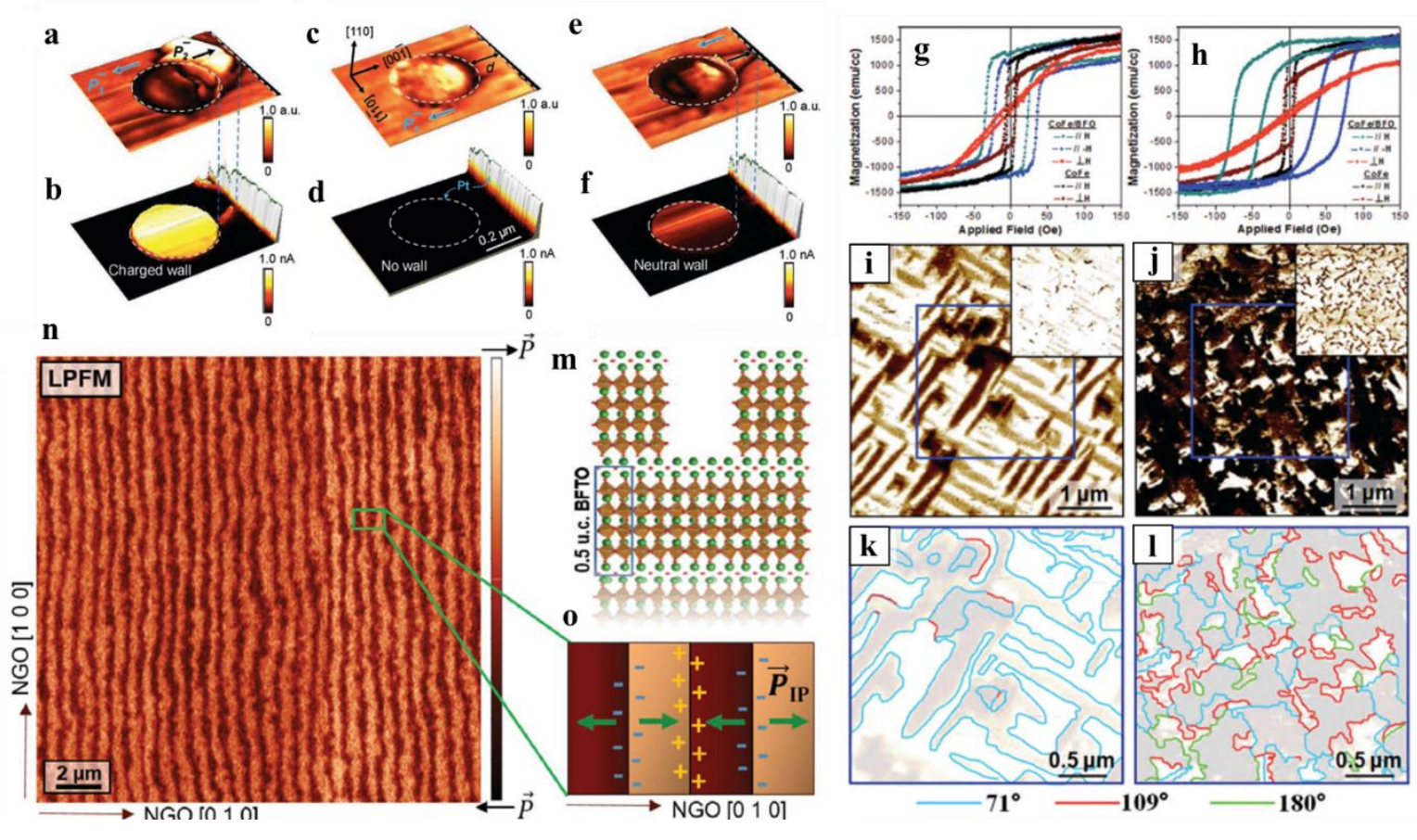

FIGURE 6| (a-f) Tristate conformational domain wall switch in $\mathrm{BiFeO}_{3}$. Lateral PFM amplitude $(\mathrm{a}, \mathrm{c}, \mathrm{e})$ and the corresponding c-AFM images $(b, d, f)$ : in the absence of domain walls $(a, b)$ and in the presence of neutral $(c, d)$, and charged (e,f) domain wall achieved with the device. Reproduced with permission from [189]. (g-l) Exchange bias dependence on domain wall type in $\mathrm{BiFeO}_{3}$. Magnetic measurements on $\mathrm{BiFeO}_{3} / \mathrm{CoFe}$ heterostructures exhibiting enhanced coercivity (g) and exchange bias (h). (i,j) In-plane and out-of-plane (inset) PFM contrast for typical $\mathrm{BiFeO}_{3}$ films corresponding to $(\mathrm{g})$ and $(\mathrm{h})$, respectively. Detailed domain wall analysis for (k) stripe-like and (I) mosaic-like $\mathrm{BiFeO}_{3}$ films. $109^{\circ}$ domain walls are directly related to exchange bias, while other domain walls still enhance coercivity. Reprinted with permission from [112]. Copyright (c) 2008, American Chemical Society. Copyright 2019 Wiley-VCH Verlag GmbH \& Co. KGaA. (m-o) Nominally charged domain walls in ultrathin BisFeTi $\mathrm{T}_{3} \mathrm{O}_{15}$ Aurivillius films (film structure depicted in $\mathrm{m}$ ). Lateral PFM contrast $(\mathrm{n}$ ) recorded for a half-unit-cell film revealing nominally charged head-to-head and tail-to-tail domain walls, sketched in a schematic (o). Reproduced with permission from [30]. Copyright 2020 Wiley-VCH Verlag GmbH \& Co. KGaA.

\section{Multiferroic heterostructures for spintronics}


The coupling between electric and magnetic orders in magnetoelectric and multiferroic heterostructures opens pathways to energy efficient spintronic devices and other novel devices (such as sensors, motors, voltage-controlled RF devices, and reconfigurable electronics) that leverage the electric to magnetic transduction. Over the past 20 years, the embodiment of devices that employ magnetoelectrics or multiferroics has grown from a magnetic memory element to reconfigurable memoryin-logic devices and circuit architectures. Here we will focus on the modern spin-based device concepts that integrate magnetoelectric multiferroic heterostructures and briefly discuss the evolution of the concepts.

\subsection{Magnetoelectric/multiferroic memory}

The magnetic random-access memory was one of the earliest devices identified where integration of a magnetoelectric or multiferroic could improve performance. This would occur through a reduced write energy via magnetoelectric switching or a multi-state output through independent control of the electric and magnetic polarizations. Here we will focus on the former as it has had the most impact on devices to date.

Spin-based magnetic random-access memory (MRAM) technology uses spin-transfer or spin-orbit torques to convert an electric charge current into a spin current that transfers angular momentum to the magnetization of the free layer in a memory element. From an energy perspective, the conversion of charge to spin current is energy inefficient as the current densities to drive switching are large ( $10^{6}-10^{7} \mathrm{~A}$ $\left.\mathrm{cm}^{-2}\right)$ and losses from resistive heating $\left(I^{2} \mathrm{R}\right)$ are significant. Magnetoelectric switching mitigates the large write currents and dissipation energy as an electric field is applied across a dielectric insulator. The energy dissipation is then dominated by charging of the capacitor or ferroelectric switching and the switching of the magnetization.

For example, the state-of-the-art spin-orbit torque (SOT) switching of a ferromagnetic layer employed low-resistivity AuPt alloys and demonstrated switching at a current density of $1.2 \times 10^{7} \mathrm{~A} / \mathrm{cm}^{2}$. The low resistivity $(80 \mu \Omega \mathrm{cm})$ and high spin Hall conductivity $\left(4.4 \times 10^{5} \Omega^{-1} \mathrm{~m}^{-1}\right)$ leads to a power dissipation per write operation that is 10 times lower than that using W [219]. Follow-up work has shown that in the $\mathrm{Au}_{0.25} \mathrm{Pt}_{0.75}$ system has reported sub-50 fJ dissipation $\left(\sim 580 \mu \mathrm{J} \mathrm{cm}^{-2}\right)$ at 1 ns current pulses and less than $10^{-}$ ${ }^{5}$ error rate [220]. In contrast, the electric field control of magnetism in rather unoptimized multiferroic heterostructures have shown energy dissipations in the range of 1-500 $\mu \mathrm{J} \mathrm{cm}^{-2}$ [49]. The primary energy 
dissipation comes from the switching of the ferroelectric polarization, or the charging of and leakage in the capacitor in the case of non-ferroelectric magnetoelectrics. Thus, the most immediate embodiment of a magnetoelectric memory element uses the magnetoelectric switching of a layer in a giant magnetoresistance (GMR) or magnetic tunnel junction (MTJ) element, whereby a small current can be used to readout the state of the device (Figure 7).

One of the first materials proposed for such applications was the room temperature magnetoelectric multiferroic $\mathrm{BiFeO}_{3}$. While the idea was put forward in the 2000 's $[7,221]$, it was not until the mid- and late 2010's that reversible magnetoelectric switching of a GMR element was demonstrated at room temperature (shown in Figure 7) $[97,222]$. By exchange coupling the free layer of the GMR stack to the $\mathrm{BiFeO}_{3}$ layer, the free layer then becomes controlled by the applied electric field to the $\mathrm{BiFeO}_{3}$. This is observed in current in-plane resistance measurements of the GMR structure versus applied voltage (Figure 7). Notably, the hysteresis and coercive voltages in the ferroelectric response of the $\mathrm{BiFeO}_{3}$ agree well with the hysteresis observed the GMR device, demonstrating a clear coupling between electrical and magnetic parameters. In the devices that involve switching the ferroelectric order parameter, the energy dissipation is dominated by that contribution. The energy dissipation (per unit area) can then be approximated by $2 \mathrm{P}_{\mathrm{S}} \mathrm{V}_{\mathrm{c}}$ where $\mathrm{P}_{\mathrm{S}}$ is the saturation polarization of the ferroelectric and $\mathrm{V}_{\mathrm{C}}$ is the coercive voltage. Thus, the energy dissipation for these pioneering structures is $\sim 450 \mu \mathrm{J} \mathrm{cm}^{-2}[49,97]$.

While the pioneering devices have energy dissipation per unit area that is comparable to the leading SOT devices, there are clear routes to tune the magnetoelectric energy landscape for improving performance. La substitution in $\mathrm{BiFeO}_{3}$ systematically destabilizes the ferroelectric order thereby reducing $P_{s}$ and $V_{C}$. Yet recent work has found that the substitution progressively increases the degeneracy of the potential energy landscape of the system and rotates the polar axis $[113,123]$. While the magnetoelectric coupling seems preserved, the increased complexity in ferroelectric and antiferromagnetic domain states compared to the undoped $\mathrm{BiFeO}_{3}$ films demands further investigation. 

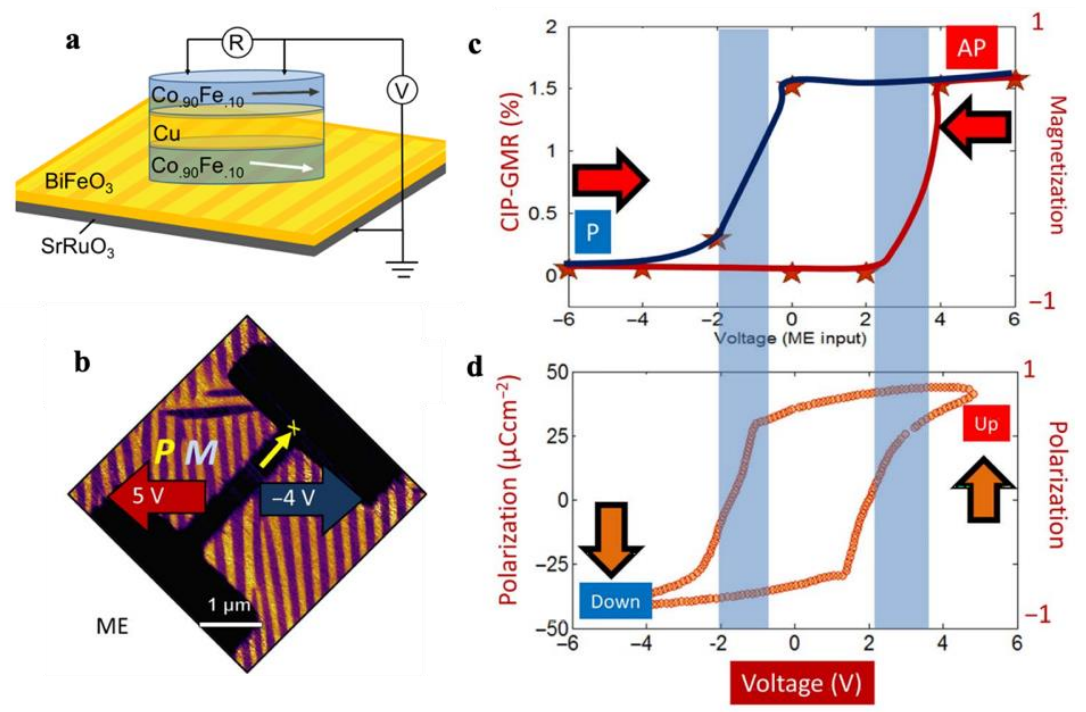

FIGURE 7| (a-d) Electric field control of a GMR device using $\mathrm{BiFeO}_{3}$. (a) Schematic of the magnetoelectric memory element. A voltage is applied across the multiferroic $\mathrm{BiFeO}_{3}$ which controls the magnetization state of the exchange coupled layer. Current is applied in the plane of the GMR stack for readout. Reprinted with permission from [223]. (b) PFM image of an actual device with the current bar oriented at $\sim 45^{\circ}$ from the $\mathrm{BiFeO}_{3}$ domains. (c,d) Measured GMR (c) and ferroelectric polarization (d) versus voltage applied across the $\mathrm{BiFeO}_{3}$ layer. The resistance of the GMR device (c) is hysteretic, non-volatile, and with coercive voltages in agreement with that from the ferroelectric switching (d) revealing the magnetoelectric switching of the $\mathrm{BiFeO}_{3}$ and the reversal of the exchange coupled magnetic layer in the GMR stack. Reprinted with permission from [222].

Recently, the field of antiferromagnetic spintronics has garnered attention due to the new physical insights, probe development of the otherwise inert state, and the new avenues for device implementation. For electrical insulators, the longitudinal and Hall resistance of a spin Hall metal in contact with the antiferromagnetic insulator will depend on the orientation of the Néel vector. This occurs either through spin Hall magnetoresistance or a proximity induced Hall effect. Work by Kosub and colleagues [107] demonstrated a purely antiferromagnetic magnetoelectric memory in a $\mathrm{Cr}_{2} \mathrm{O}_{3} / \mathrm{Pt}$ heterostructure (Figure 8). Here the Pt layer couples to the surface magnetization state of $\mathrm{Cr}_{2} \mathrm{O}_{3}$, which can be readout through a Hall measurement. The surface magnetization state is determined by the antiferromagnetic domain state which can be switched by simultaneous application of an electric and magnetic field with a critical electric and magnetic field product [105]. A few features of this purely antiferromagnetic magnetoelectric memory are particularly noteworthy. First, contrary to the $\mathrm{BiFeO}_{3}$ based devices discussed previously, the absence of an exchange coupled ferromagnetic layer is expected to decrease the device switching times due to the 
faster spin dynamics in antiferromagnetic systems. Second, $\mathrm{Cr}_{2} \mathrm{O}_{3}$ is not ferroelectric, therefore, energy dissipation is capacitive and ultra-low $\left(\sim 6 \times 10^{-2} \mu \mathrm{J} \mathrm{cm}^{-2}-\right.$ assuming ideal dielectric behavior and neglecting the dissipation associated with the applied 6.3 kOe magnetic field [49]). Finally, extending the idea of coupling to the surface magnetization to quantum materials such as topological insulators, topological ferromagnetic insulators, or spin-valley Hall materials opens doors to electrical control spin-momentum locked phenomena $[224,225]$.

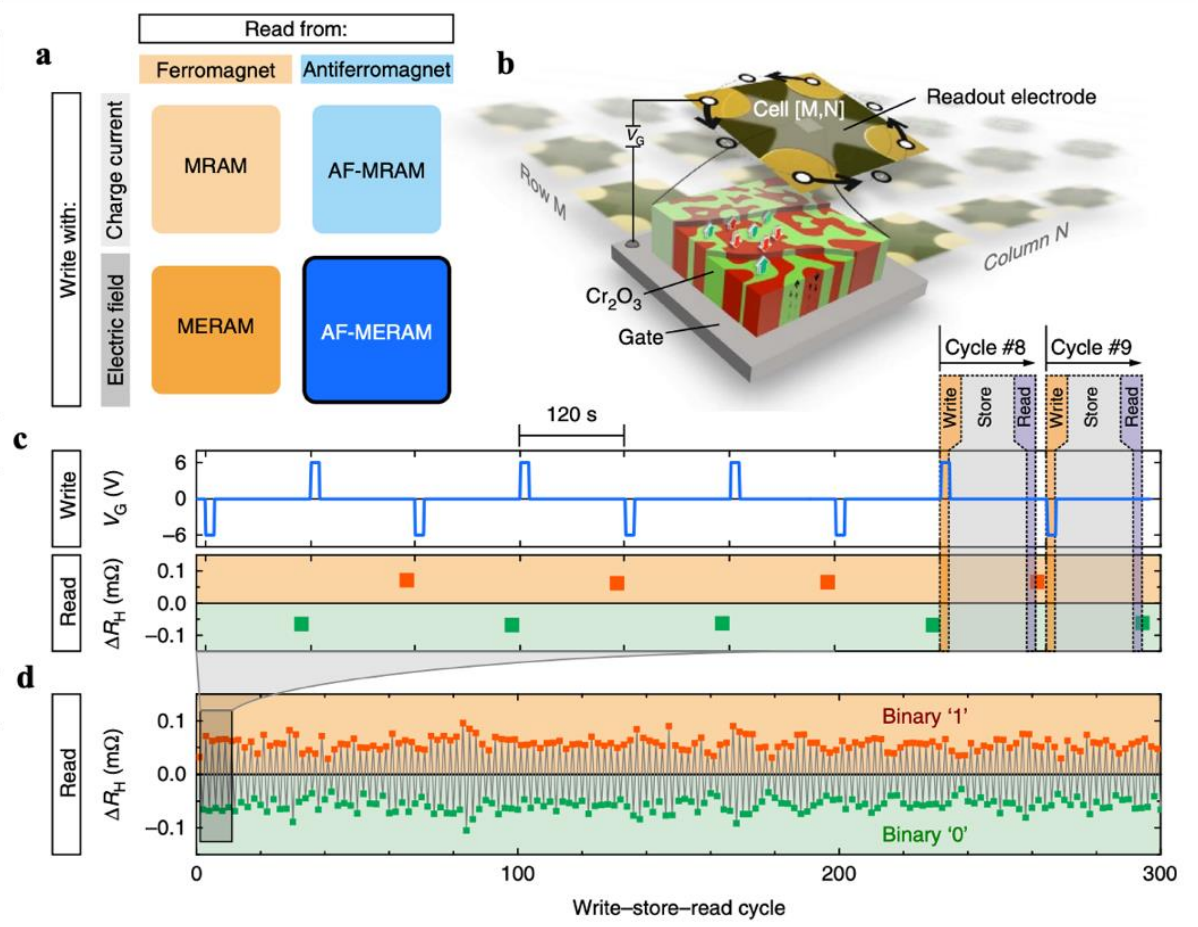

FIGURE 8| (a-d) $\mathrm{Cr}_{2} \mathrm{O}_{3}$ based antiferromagnetic magnetoelectric memory. (a) Schematic of spin-based memory elements categorized by the writing stimulus and order parameter for readout. (b) The surface magnetization of antiferromagnetic magnetoelectric $\mathrm{Cr}_{2} \mathrm{O}_{3}$ enables readout of the antiferromagnetic state of a bit through a Hall effect in an adjacent Pt layer. (c,d) Write voltage and Hall signal showing the fundamental write-read procedure (c) and robustness of the device with cycle number (d). Reprinted with permission from [107]. Copyright (C 2017, Springer Nature.

\subsection{Magnetoelectric/multiferroic Logic}

Computation based on complimentary metal-oxide-semiconductor (CMOS) transistors is inherently inefficient due to the volatility of the device and the fundamental losses as the devices are scaled down. In the search for a new materials platform for the next generation of computation, non- 
volatile technologies have emerged as promising avenues as the total energy dissipation, write energy, and scalability can be shown to outperform CMOS. Work led by Intel and researchers at UC Berkeley have proposed a Magneto-Electric-Spin-Orbit (MESO) logic as one such technology (Figure 9) [76]. The fundamental device operates with a magnetoelectric write operation that determines the magnetization orientation of a magnetic bit. A supply current injects electrons into the magnetic bit and spin-polarizes them. This spin polarized current is then injected into a spin-orbit layer that uses the interface RashbaEdelstein effect or the inverse spin Hall effect to create a transverse current that is used for readout or cascading logic devices.

At the heart of the device is the magnetoelectric write operation which takes the voltage input and controls the orientation of the magnetization which is hysteretic, non-volatile, and 10 to 1000 times more energy efficient than SOT or STT switching [49]. Critical for applications is the efficiency of the device at scale. The authors, using accepted bulk and thin film material parameters, find that MESO will operate at 1-10 aJ dissipation and sub-100 mV per operation at $20 \times 30 \mathrm{~nm}^{2}$ nanomagnet and $10 \times 10 \times 10 \mathrm{~nm}^{3}$ magnetoelectric dimensions. Proposed MESO logic circuits compare favorably to CMOS fanout-4 inverter, two-input NAND adder, 32-bit ripple-carry adder and 32-bit ALU logic circuits. Particularly when compared to the inverter in 2018, MESO shows a 10-100 fold switching energy reduction in a non-volatile technology. 

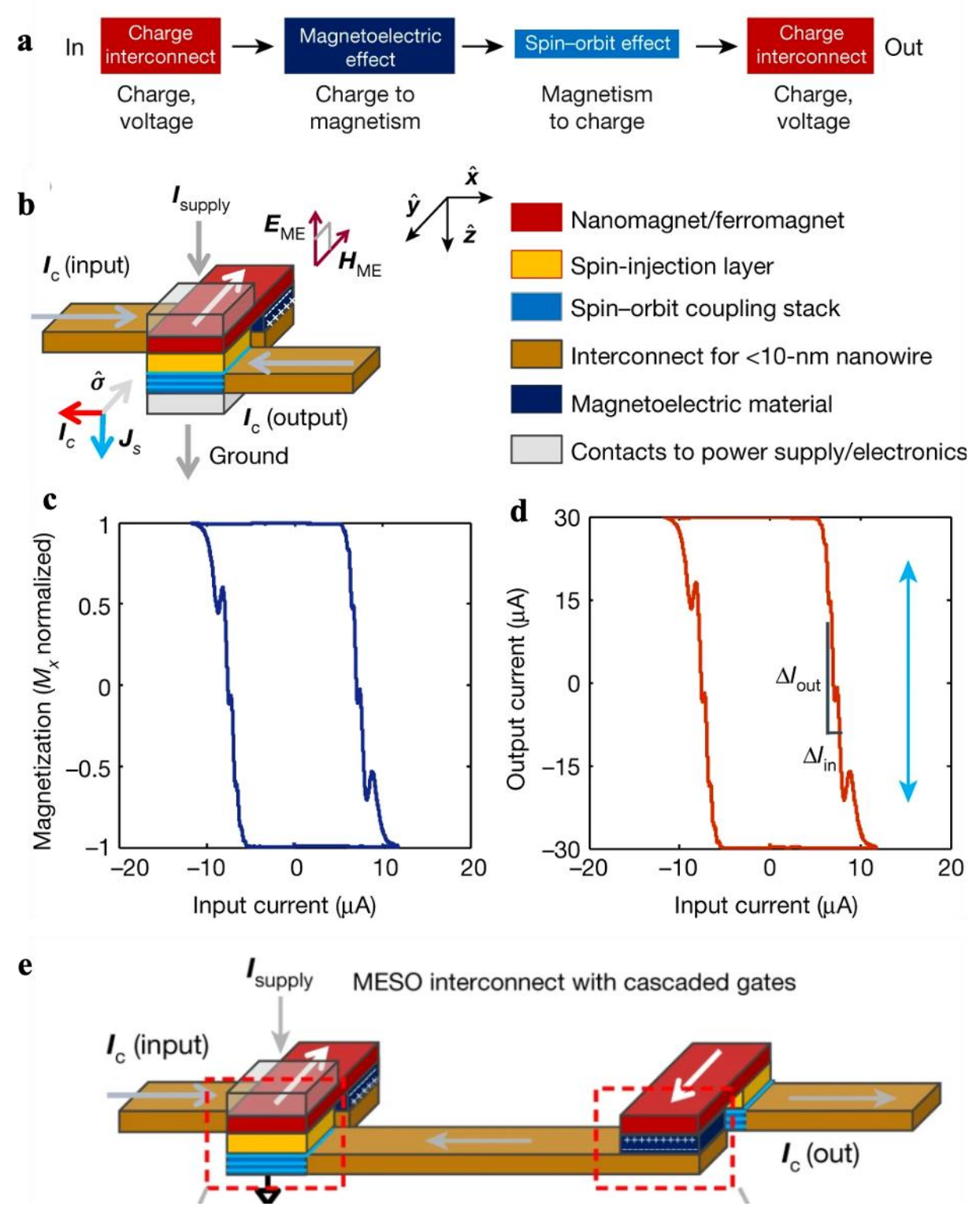

FIGURE 9| (a-e) The MESO device and connectivity. (a) Shows the fundamental mechanisms employed in the MESO devices from an input signal to output. (b) Schematic of the MESO device. Input, output, supply and spin currents are

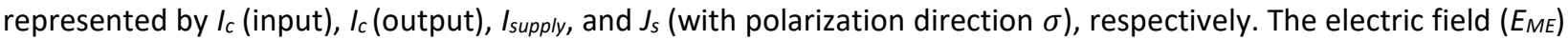
applied to the magnetoelectric at the input and the effective magnetic field $\left(H_{M E}\right)$ generated that couples to the ferromagnetic bit are shown. (c,d) Normalized magnetization direction (c) and output current (d) versus input current. The output is hysteretic and non-volatile. The output current is proportional to the orientation of the magnetization which is controlled by the input to the magnetoelectric layer. (e). Schematic showing the connectivity to form logic structures. Reprinted with permission from [76]. Copyright @ 2018, Springer Nature.

The estimations are impressive, and the race is on to realize such a device. Yet, several materials challenges remain to be tackled. While great progress has been made to realize the electrical switching of 
magnetism, demonstrating this at technologically relevant scale and solving defect pinning at metal-oxide interfaces remain largely untackled. The observation of application-compatible spin Hall resistivities in material systems compatible with oxide magnetoelectrics has been unexplored.

\subsection{Neuromorphic devices}

Biologically inspired computing mimics the neural structure of the brain and is highly efficient at difficult tasks. Of current interest is the development of neuromorphic circuits that are able to analyze videos and images for recognition and filtering applications. While such neural networks can be realized using CMOS technology, the inherent inefficiencies of CMOS devices leave room for non-volatile beyondCMOS technologies to enhance the performance and parallelization. Spin-based neurons have been proposed and simulated. While showing promise, many rely on magnetic tunnel junctions, which makes readout challenging due to the nature of resistance and spin Hall and spin torques which are unideal due to resistive heating [226].

Building on the MESO device, simulations of a cellular neural network with inverse RashbaEdelstein magnetoelectric (IRME) neurons and CMOS synapses performing a low-pass filtering have been reported to be fast and efficient [227]. In this case, the IRME neuron state is written with a voltage across the antiferromagnetic magnetoelectric layer, which controls the magnetization state of an exchange coupled ferromagnetic layer. Readout is done with a supply current that is spin polarized by the ferromagnet and injected in the inverse Rashba-Edelstein heterostructure, which converts the injected spin current into a transverse voltage. Assuming materials parameters for CoFe as the ferromagnet, magnetoelectric coefficients comparable to $\mathrm{BiFeO}_{3}$, and an inverse Rashba-Edelstein parameter for that found in $\mathrm{LaAlO}_{3} / \mathrm{SrTiO}_{3}$ polar interface [228], the IRME performs state transitions in $\approx 100 \mathrm{ps, \text {network }}$ operations with a per-neuron energy cost on the order of $\approx 1 \mathrm{fJ}$, and is non-volatile [227]. The IRME device has several notable features that lead to enhanced performance. Beyond the energy efficiency of the IRME neuron and its non-volatility which are enabled by the materials, the IRME network is parallelized as each neuron operates simultaneously and performs both write and read operations at the same time.

\subsection{Hybrid magnetoelectric-spin-orbit torque heterostructures}

Composite magnetoelectric multiferroic heterostructures are relatively easy to realize and 
demonstrate large magnetoelectric effects at and above room temperature. While such heterostructures have displayed robust coupling and even $90^{\circ}$ switching of the magnetization, a full magnetization reversal is often desired to maximize readout signal. Thus, hybrid magnetoelectric-spin-orbit torque heterostructures have emerged to mitigate the weakness of SOT and composite magnetoelectric switching and leveraging their respective strengths. One embodiment of this concept is the hybrid magnetoelectricspin-orbit torque (ME-SOT) heterostructure, which consists of a composite magnetoelectric with a spinorbit torque active layer.

The main steps to switching in such a heterostructure first involve a voltage applied to the piezoelectric layer to drive the $90^{\circ}$ switching of the magnetization. Then a current is applied to the SOT layer to drive the remaining $90^{\circ}$ switch. Simulations of this two-step hybrid switching process indicate that the current pulse width for reliable spin-orbit torque switching may decrease by a factor of 10-1000, depending on the anisotropy and damping parameter of the magnetostrictive layer [119]. The faster switching times is the result of the reduced processional motion of the magnetization as the magnetoelectric has already initialized it to the peak of the magnetic anisotropy barrier (assuming a uniaxial anisotropy). The device demonstrates some key advantages, in particular high temperature operation, simple materials synthesis, deterministic $180^{\circ}$ magnetization switching at a $10-1000$ times improved energy-delay performance.

Other manifestations of hybrid ME-SOT heterostructures have been explored to break critical symmetries and lift degeneracies in SOT switching [229-232]. For instance, SOT switching of a perpendicular magnetic anisotropy (PMA) magnet by the spin Hall effect of isotropic heavy metals is complicated by non-deterministic switching. The symmetry of the system can be changed to realize deterministic switching by applying an in-plane magnetic field, switching in the presence of an in-plane exchange bias [232], but also by magnetoelectrically induced changes to the magnetic anisotropy and electric field gradients $[233,234]$.

\section{Future perspectives and concluding remarks}

The current achievements dealing the electric field control of magnetism have been reviewed. Let us now address one of the main challenges limiting the insertion of multiferroics into spintronic devices, i.e. the determination of the magnetoelectric switching dynamics. Switching dynamics are typically estimated from Landau-Lifshitz-Gilbert-Slonczewski (LLGS) simulations and have seen little experimental 
work. Therefore, despite all the progress, the competitiveness of magnetoelectric-based devices with respect to established spin current based technology is still under debate. We review emergent probes of the dynamic of multiferroic antiferromagnetic states in thin film heterostructures. We finally present some new avenues in the field, highlighting the potential of light to probe and even assist multiferroic switching.

\subsection{Magnetoelectric switching dynamics}

Magnetoelectric random-access memories or magnetoelectric spin-orbit logic devices with strong charge-to-spin coupling promise attojoule switching energies. If those concepts shall become reality, the scientific community needs to intensify efforts towards a deeper understanding of multiferroic and interlayer exchange dynamics. The lack of existing data on multiferroic switching dynamics partly originate from the difficulty to simultaneously and operando probe two order parameters, i.e. electric polarization and net magnetization. The direct observation of the ferroelectric-ferromagnetic domain correlation during a magnetoelectric switching event is a prerequisite to the understanding of the switching mechanism and its dynamics.

In the model system, $\mathrm{BiFeO}_{3} /$ ferromagnetic heterostructures, the electric field induced magnetization reversal involves a two-step switching process. Within the unit cell, the polarization projection onto the interface plane rotates twice by $90^{\circ}$ [97]. The resulting $180^{\circ}$ reversal of the polarization projection and the corresponding antiferromagnetic configuration in $\mathrm{BiFeO}_{3}$ in the final state thus leads to the $180^{\circ}$ switch of the exchange coupled magnetization in the ferromagnetic layer $[97,102]$. While the ferroelectric switching has been unambiguously demonstrated, the dynamics of the magnetic order in $\mathrm{BiFeO}_{3}$ and in the magnetically coupled ferromagnetic layer have remained unexplored.

On the one hand, several experimental techniques allow probing of the ferroelectric switching and domain dynamics, even in a buried state. Beyond the investigation of the remnant hysteresis using positive-up negative-down (PUND) method, hard X-ray diffraction [235,236], hard X-ray photoemission spectroscopy [237], second harmonic generation [177,238], X-ray photon correlation spectroscopy [239], and piezoresponse force spectroscopy $[126,240]$ provide direct access to the polarization with time resolution and are compatible with operando analysis. On the other hand, antiferromagnetic states, dominating the magnetoelectric switching mechanism, lack a net magnetization and have therefore remained difficult to address. Over the last few years, the following techniques have emerged as efficient probe of antiferromagnetic state in thin film heterostructures. 
The single-spin magnetometer scanning probe technique - Based on a point-like impurity nitrogen-vacancy (NV) defect in diamond mounted on a scanning tip [241-243], this technique provides an outstanding magnetic sensitivity (down to a few femtotesla) with a spatial resolution comparable to the one achieved with atomic force microscopy. It has been used to spatially resolve the local magnetic order in multiferroic antiferromagnetic $\mathrm{BiFeO}_{3}$ thin films [244]. The periodic modulation in the magnetic response corresponds to the spin cycloid in the films. Such a measurement allows the correlation of the antiferromagnetic domain state with the ferroelectric domain architecture obtained by PFM.

Magnetoelectric force microscopy (MeFM) - The MeMFM is another scanning probe based technique. A magnetically tip is used to probe the magnetoelectric response of the material exposed to an AC voltage. It is based on the lock-in detection of the magnetic force microscopy signal from the electric-field-induced magnetization in magnetoelectric materials. First developed in order to probe magnetoelectric domains in hexagonal $\mathrm{ErMnO}_{3}$ crystals at low temperature [245], its efficiency to monitor the magnetoelectric response of the $\mathrm{Cr}_{2} \mathrm{O}_{3}$ crystal model system has been recently demonstrated [246], see Figure 10a-b. The MeFM requires the magnetoelectric material to be integrated in a capacitor to trigger the magnetoelectric response. On one hand, such capping may prevent simultaneous piezoresponse based ferroelectric domain imaging. On the other hand, the use of heavy metals for the electrode might provide a platform for the investigation of hybrid magnetoelectric STT, SOT type of heterostructures, see section 4.4.

Optical second harmonic generation (SHG) - SHG can be sensitive to the reduction of symmetry through the magnetic ordering of spins. We refer the reader to several reviews dealing with the potential of SHG to probe ferroic states in bulk and thin film materials $[238,247,248]$. Here we highlight the seminal demonstration in antiferromagnetic magnetoelectric $\mathrm{Cr}_{2} \mathrm{O}_{3}$ crystal, in which the SHG was employed to image antiferromagnetic domains [140], see Figure 10c-d. Such an optical based technique further offers time resolution matching the ultrafast dynamics of the ferroic switching speed. The potential of timeresolved SHG to determine antiferromagnetic ultrafast dynamics has recently been revealed [249]. In bulk $\mathrm{YMnO}_{3}$, the spin ordering modulation upon coherent magnon excitation could be tracked using a combination of Faraday rotation and SHG. In multiferroic thin films systems, SHG has been used to identify the antiferromagnetic domains in epitaxially strained $\mathrm{BiFeO}_{3}$ [250]. Furthermore, taking advantage of the optical rectification process in intense light fields, $100 \mathrm{fs}$ light pulses were used to generate electric pulses in the antiferromagnetic resonance regime of $\mathrm{BiFeO}_{3}$. The corresponding change in the antiferromagnetic domains were imaged by SHG after the optically induced electric pulses, see Figure 10e-f. This work suggests new avenues towards the optical control of multiferroic and magnetoelectric switching. We address the non-invasive optical control of such switching events in the next section. 

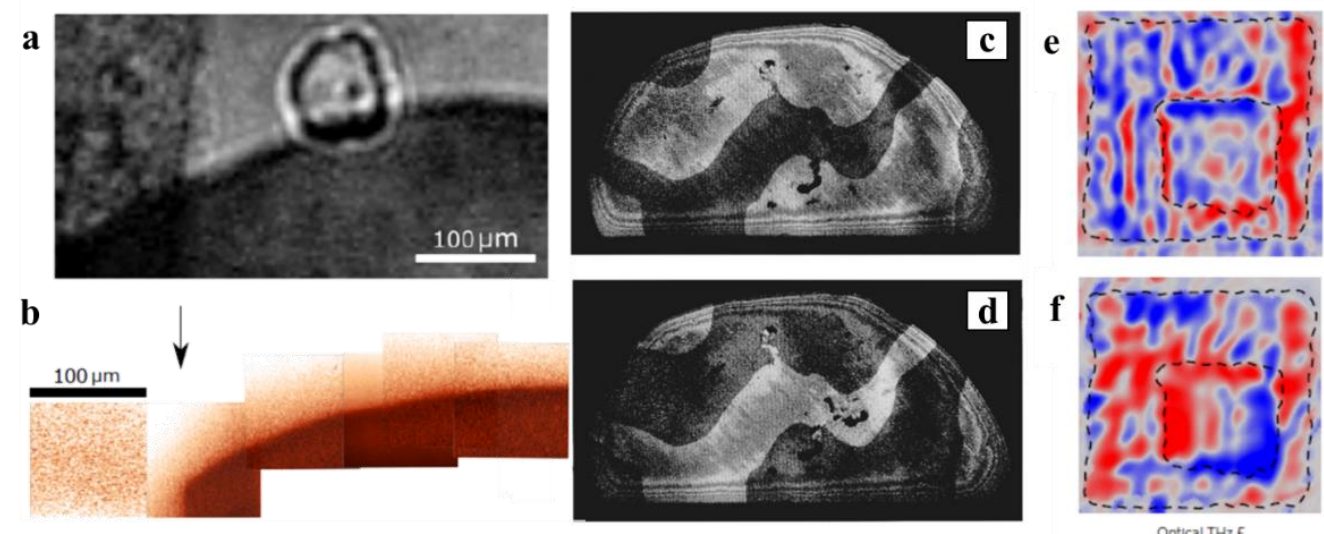

FIGURE 10| (a-b) $\mathrm{Cr}_{2} \mathrm{O}_{3}$ crystal imaged using optical second harmonic (a) and magnetoelectric force microscopy (b). Reprinted with permission from [246]. (c-d) Optical second harmonic generation images with right (c) and (d) left circularly polarized light. The sample is $6 \mathrm{~mm}$ long. Reprinted with permission from [140]. Copyright 1995, AIP Publishing LLC. (e-f) Optical second harmonic generation based reconstruction of the antiferromagnetic configurations of the patterns in $\mathrm{BiFeO}_{3}$ thin films in virgin state (e) and after sub-coercive picosecond electric pulses delivered by rectification of intense laser pulses (f). Reprinted with permission from [250]. Copyright @ 2017, Springer Nature.

\subsection{Towards all optical magnetoelectric switching}

Light, which has been mainly used to probe materials $[247,248]$, now appears to act on ferroic states. Its potential to induce magnetization reversal in thin films was recently demonstrated [251,252]. The long working distance and ultrafast timescale of optical processes render it further compatible with device integration. The light-matter interaction in ferroelectrics however drastically differs from the ferromagnetic analogs. The light induced processes in ferroelectric materials span from local heating [253] to photo-induced flexoelectric effect [254], photovoltaic effect [255] or photostriction [256]. In particular, photo-induced charge carriers may migrate towards domain walls or to the film surface and act on the bound charge screening at the ferroelectric surfaces. Considering the depolarizing impact on domain state in epitaxial thin ferroelectric films, see section 3 and $[46,144]$, light irradiation on thin films may therefore emerge as an efficient approach towards the domain modulation in ferroelectric and multiferroic magnetoelectric systems.

A pioneering work dealing with light-induced polarization changes investigations focused mainly on bulk materials and led to local domain wall motions or phase transitions [255,257-259]. The direct monitoring of an optically induced domain wall motion and domain configuration change in ferroelectric material opened new avenues for tuning macroscopic polarization states by means of a non-contact 
external control. Using in-situ XRD measurements, the $\mathrm{BaTiO}_{3}$ in-plane oriented a-domains reorient in an orthogonal axis point either in-plane or out-of-plane upon irradiation under polarized light as shown in Figure 11a-c. The process is fully reversible and the domain state returns to its original configuration in the dark.

In highly strained multiferroic $\mathrm{BiFeO}_{3}$ films, the flexophotovoltaic effects [260], combining strain gradient and light sensitivity has been revealed as a potential degree of freedom for all optical multiferroic magnetoelectric switch. The strain induced morphotropic boundary in $\mathrm{BiFeO}_{3}$ films grown on highly compressive epitaxial strain leads to a rhombohedral and tetragonal phase coexistence. Upon illumination with a coherent light source, the balance between the two phases can be controlled, as illustrated in Figure $11 \mathrm{~d}$-e. The mechanism involves a combination of flexoelectricity and thermal heating. Most importantly, the first experimental data further indicate that the light induced changes in the film domain state are accompanied by an alteration of the antiferromagnetic state.

Finally, photocurrents generated via the bulk photovoltaic may be engineered in order to achieve local ferroelectric switching event. Using a tip enhanced electric field created by increased photocurrent density at the tip, the light irradiation induces an electric field surpassing the coercive electric field, as shown in Figure 11f-g. The bulk photovoltaic effect depends on the crystallographic direction and on the light polarization such that an all-optical light polarization dependent switching can be achieved in multiferroic $\mathrm{BiFeO}_{3}$ films by simply changing the light polarization. Because strain strongly impacts the photocurrent generation [261], the recent development in epitaxial design might further support the development of optically induced functionality in magnetoelectric multilayers.
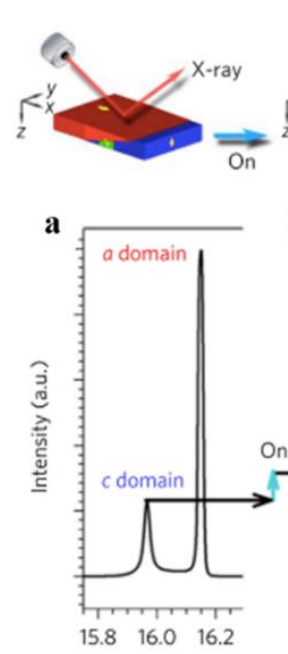

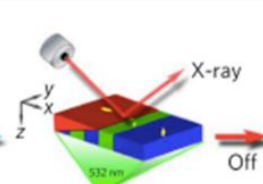
$\mathbf{b}$

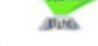

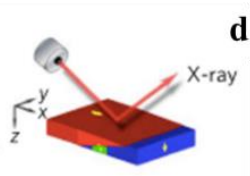
c

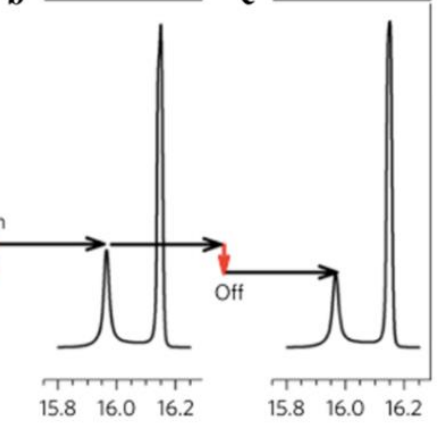

$$
2 \theta(\mathrm{deg})
$$

f d
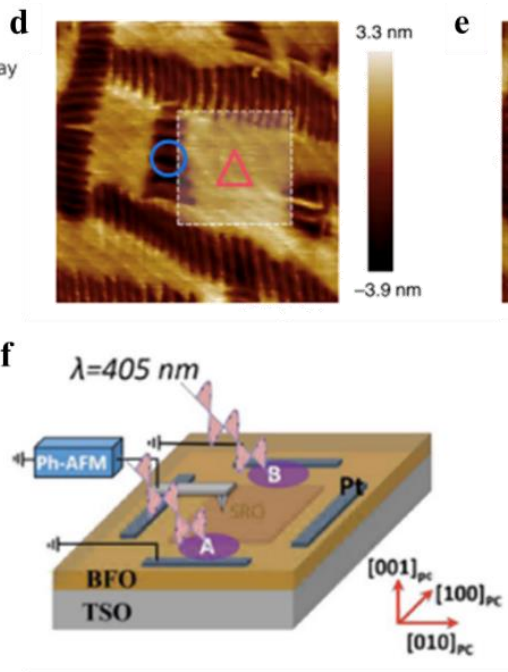
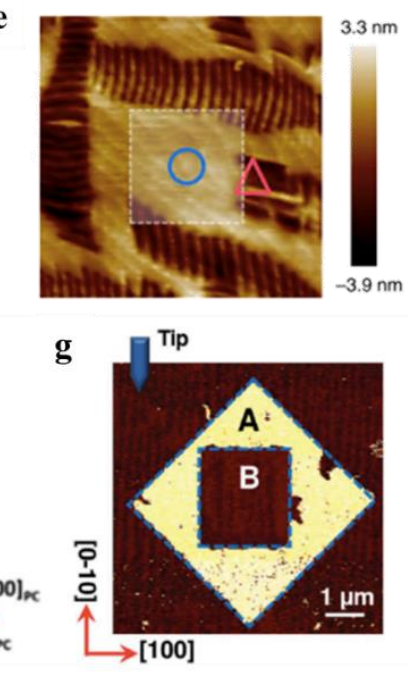
FIGURE 11| (a-c) Reversible optical control of macroscopic polarization in $\mathrm{BaTiO}_{3}$. Sequence of synchrotron radiation high-resolution XRD patterns of (002)/(200) reflections corresponding to the off (a)-on (b)-off (c) light succession. The contribution of the $c$ domains enhances when the light is on as a consequence of light-induced domain wall motion and is reversible. Reprinted with permission from [258]. Copyright (C) 2017, Springer Nature. (d-e) Deterministic optical control of phase distribution in mixed-phase $\mathrm{BiFeO}_{3}$. Topography images of mixed-phase $\mathrm{BiFeO}_{3}$ after illumination of areas marked with a triangle (d) and circle (e). Reprinted with permission from [254]. Copyright (C) 2019, Springer Nature. (f-g) Light-induced reversible switching of ferroelectric $\mathrm{BiFeO}_{3}$ polarization via tuning illumination area. (f) Schematic showing photoelectric atomic force microscopy (Ph-AFM) setup and illumination areas resulting in photocurrent flow in different directions and hence reversed electric-field polarity under the tip leading to a reversible switch shown in PFM image (g). Reproduced with permission from [255]. Copyright 2018 Wiley-VCH Verlag GmbH \& Co. KGaA)

\section{Conclusion}

In this article, we reviewed the most recent developments in the field of magnetoelectric heterostructures. More complete review articles dedicated to artificial multiferroic heterostructures $[4,5]$ or domain manipulation $[45,262]$ can be found elsewhere. Magnetoelectric heterostructures have first emerged as an alternative to the scarcity of single-phase multiferroic magnetoelectric materials [8]. They now play a major role in the pursuit of low energy consuming devices paradigms.

New probing tools are pushing the understanding of domain coupling and domain imprint in the multilayers [2]. In particular non-invasive, operando investigations [177,238] are key towards the determination of the magnetoelectric switching dynamics.

Finally, the compatibility of magnetoelectric architecture with SOT or all optical switching schemes allows realization of hybrid devices with expected ultra-fast dynamics and ultra-low energy consumption.

\section{Acknowledgements}

E.G. and M.T. acknowledge the financial support by the Swiss National Science Foundation under project No. 200021_188414. P.B.M. and J.T.H. acknowledge that this work was supported in part by the Semiconductor Research Corporation (SRC) as the NEWLIMITS Center and NIST through award number 70NANB17H041. 


\section{References}

[1] Fiebig M, Lottermoser T, Meier D and Trassin M 2016 The evolution of multiferroics Nat. Rev. Mater. 116046

[2] Spaldin N A and Ramesh R 2019 Advances in magnetoelectric multiferroics Nat. Mater. 18 20312

[3] Ramesh R and Spaldin N A 2007 Multiferroics: progress and prospects in thin films Nat. Mater. 6 21-9

[4] Trassin M 2016 Low energy consumption spintronics using multiferroic heterostructures J. Phys. Condens. Matter 28

[5] Fernandes Vaz C A and Staub U 2013 Artificial multiferroic heterostructures J. Mater. Chem. C 1 6731-42

[6] Scott J F 2007 Multiferroic memories Nat. Mater. 6 256-7

[7] Bibes M 2008 Towards a magnetoelectric memory 7 7-8

[8] Hill N A 2000 Why Are There so Few Magnetic Ferroelectrics ? J. Phys. Chem. B 1046694

[9] Wiegelmann H, Jansen A G M, Wyder P, Rivera J P and Schmid H 1994 Magnetoelectric effect of Cr2O3 in strong static magnetic fields Ferroelectrics 162 141-6

[10] Fiebig M, Fröhlich D, Krichevtsov B B and Pisarev R V 1994 Second Harmonic Generation and Magnetic-Dipole-Electric-Dipole Interference in Antiferromagnetic Cr2O3 Phys. Rev. Lett. 73 2127-30

[11] Van Aken B B, Palstra T T M, Filippetti A and Spaldin N A 2004 The origin of ferroelectricity in magnetoelectric YMnO3 Nat. Mater. 3 164-70

[12] Fiebig M, Lottermoser T, Fröhlich D, Goltsev A V. and Pisarev R V. 2002 Observation of coupled magnetic and electric domains Nature 419 818-20

[13] Bertaut E F and Mercier M 1963 Structure Magnetique de MnYO3 Phys. Lett. 5 27-9

[14] Nordlander J, Campanini M, Rossell M D, Erni R, Meier Q N, Cano A, Spaldin N A, Fiebig M and Trassin M 2019 The ultrathin limit of improper ferroelectricity Nat. Commun. 105591 

M 2012 Anisotropic conductance at improper ferroelectric domain walls Nat. Mater. 11 284-8

[16] Schaab J, Skjærvø S H, Krohns S, Dai X, Holtz M E, Cano A, Lilienblum M, Yan Z, Bourret E, Muller D A, Fiebig M, Selbach S M and Meier D 2018 Electrical half-wave rectification at ferroelectric domain walls Nat. Nanotechnol. 13 1028-34

[17] Evans D M, Holstad T S, Mosberg A B, Småbråten D R, Vullum P E, Dadlani A L, Shapovalov K, Yan Z, Bourret E, Gao D, Akola J, Torgersen J, van Helvoort A T J, Selbach S M and Meier D 2020 Conductivity control via minimally invasive anti-Frenkel defects in a functional oxide Nat. Mater.

[18] Wang J, Neaton J B, Zheng H, Nagarajan V, Ogale S B, Liu B, Viehland D, Vaithyanathan V, Schlom D G, Waghmare U V, Spaldin N A, Rabe K M, Wuttig M and Ramesh R 2003 Epitaxial BiFeO3 multiferroic thin film heterostructures. Science 299 1719-22

[19] Zhang J X, He Q, Trassin M, Luo W, Yi D, Rossell M D, Yu P, You L, Wang C H, Kuo C Y, Heron J T, Hu Z, Zeches R J, Lin H J, Tanaka A, Chen C T, Tjeng L H, Chu Y H and Ramesh R 2011 Microscopic origin of the giant ferroelectric polarization in tetragonal-like BiFeO3 Phys. Rev. Lett. 107 1-5

[20] Lebeugle D, Colson D, Forget A, Viret M, Bataille A M and Gukasov A 2008 Electric-field-induced spin flop in BiFeO3 single crystals at room temperature Phys. Rev. Lett. 100 1-4

[21] Lebeugle D, Mougin A, Viret M, Colson D and Ranno L 2009 Electric field switching of the magnetic anisotropy of a ferromagnetic layer exchange coupled to the multiferroic compound BiFeO3 Phys. Rev. Lett. 103257601

[22] Sando D, Barthélémy A and Bibes M 2014 BiFeO3 epitaxial thin films and devices: Past, present and future J. Phys. Condens. Matter 26

[23] Heron J T, Schlom D G and Ramesh R 2014 Electric field control of magnetism using BiFeO3-based heterostructures Appl. Phys. Rev. 121303

[24] Trassin M, Viart N, Versini G, Barre S, Pourroy G, Lee J, Jo W, Dumesnil K, Dufour C and Robert S 2009 Room temperature ferrimagnetic thin films of the magnetoelectric Ga2-xFexO3 J. Mater. Chem. $198876-80$

[25] Trassin M, Viart N, Versini G, Loison J L, Vola J P, Schmerber G, Cregut O, Barre S, Pourroy G, Lee J H, Jo W and Meny C 2007 Epitaxial thin films of multiferroic GaFeO3 on conducting indium tin 
oxide (001) buffered yttrium-stabilized zirconia (001) by pulsed laser deposition Appl. Phys. Lett. $912-5$

[26] Thomasson A, Cherifi S, Lefevre C, Roulland F, Gautier B, Albertini D, Meny C and Viart N 2013 Room temperature multiferroicity in Ga0.6Fe1.403:Mg thin films J. Appl. Phys. 113 3-7

[27] Homkar S, Preziosi D, Devaux X, Bouillet C, Nordlander J, Trassin M, Roulland F, Lefèvre C, Versini G, Barre S, Leuvrey C, Lenertz M, Fiebig M, Pourroy G and Viart N 2019 Ultrathin regime growth of atomically flat multiferroic gallium ferrite films with perpendicular magnetic anisotropy Phys. Rev. Mater. 3 1-10

[28] Aurivillius B 1949 Mixed Bismuth Oxides with Layer Lattices. 1. The Structure Type of CaNb2Bi2O9 Ark. Kemi. 1 463-80

[29] Benedek N A, Rondinelli J M, Djani H, Ghosez P and Lightfoot P 2015 Understanding ferroelectricity in layered perovskites: New ideas and insights from theory and experiments Dalt. Trans. 44 10543-58

[30] Gradauskaite E, Campanini M, Biswas B, Schneider C W, Fiebig M, Rossell M D and Trassin M 2020 Robust In-Plane Ferroelectricity in Ultrathin Epitaxial Aurivillius Films Adv. Mater. Interfaces $20002021-8$

[31] Keeney L, Smith R J, Palizdar M, Schmidt M, Bell A J, Coleman J N and Whatmore R W 2020 Ferroelectric Behavior in Exfoliated 2D Aurivillius Oxide Flakes of Sub-Unit Cell Thickness Adv. Electron. Mater. 1901264

[32] Wang C, Ke X, Wang J, Liang R, Luo Z, Tian Y, Yi D, Zhang Q, Wang J, Han X F, Van Tendeloo G, Chen L Q, Nan C W, Ramesh R and Zhang J 2016 Ferroelastic switching in a layered-perovskite thin film Nat. Commun. 710636

Lee H N, Hesse D, Zakharov N and Gösele U 2002 Ferroelectric Bi3.25La0.75Ti3o12 films of uniform a-axis orientation on silicon substrates Science 296 2006-9

[34] Faraz A, Maity T, Schmidt M, Deepak N, Roy S, Pemble M E, Whatmore R W and Keeney L 2017 Direct visualization of magnetic-field-induced magnetoelectric switching in multiferroic aurivillius phase thin films J. Am. Ceram. Soc. $100975-87$

[35] Keeney L, Downing C, Schmidt M, Pemble M E, Nicolosi V and Whatmore R W 2017 Direct atomic 
scale determination of magnetic ion partition in a room temperature multiferroic material Sci. Rep. 7 1-11

[36] Baibich M N, Broto J M, Fert A, Van Dau F N, Petroff F, Eitenne P, Creuzet G, Friederich A and Chazelas J 1988 Giant magnetoresistance of (001)Fe/(001)Cr magnetic superlattices Phys. Rev. Lett. $612472-5$

[37] Binasch G, Grünberg P, Saurenbach F and Zinn W 1989 Enhanced magnetoresistance in layered magnetic structures with antiferromagnetic interlayer exchange Phys. Rev. B 39 4828-30

[38] Myers E B, Ralph D C, Katine J A, Louie R N and Buhrman R A 1999 Current-induced switching of domains in magnetic multilayer devices Science 285 867-70

[39] Jian-Gang Z H U 2008 Magnetoresistive random access memory: The path to competitiveness and scalability Proc. IEEE 96 1786-98

[40] Miron I M, Gaudin G, Auffret S, Rodmacq B, Schuhl A, Pizzini S, Vogel J and Gambardella P 2010 Current-driven spin torque induced by the Rashba effect in a ferromagnetic metal layer Nat. Mater. $9230-4$

[41] Miron I M, Garello K, Gaudin G, Zermatten P J, Costache M V., Auffret S, Bandiera S, Rodmacq B, Schuhl A and Gambardella P 2011 Perpendicular switching of a single ferromagnetic layer induced by in-plane current injection Nature 476 189-93

[42] Vélez S, Schaab J, Wörnle M S, Müller M, Gradauskaite E, Welter P, Gutgsell C, Nistor C, Degen C L, Trassin M, Fiebig M and Gambardella P 2019 High-speed domain wall racetracks in a magnetic insulator Nat. Commun. 104750

[43] Zhuravlev M Y, Sabirianov R F, Jaswal S S and Tsymbal E Y 2005 Giant electroresistance in ferroelectric tunnel junctions Phys. Rev. Lett. 94 1-4

[44] Garcia V, Fusil S, Bouzehouane K, Enouz-Vedrenne S, Mathur N D, Barthélémy A and Bibes M 2009 Giant tunnel electroresistance for non-destructive readout of ferroelectric states Nature $\mathbf{4 6 0}$ $81-4$

[45] Strkalj N, Gradauskaite E, Nordlander J and Trassin M 2019 Design and manipulation of ferroic domains in complex oxide heterostructures Materials (Basel). 12 
[46] Strkalj N, De Luca G, Campanini M, Pal S, Schaab J, Gattinoni C, Spaldin N A, Rossell M D, Fiebig M and Trassin M 2019 Depolarizing-Field Effects in Epitaxial Capacitor Heterostructures Phys. Rev. Lett. 123147601

[47] Junquera J and Ghosez P 2003 Critical thickness for ferroelectricity in perovskite ultrathin films Nature 422 506-9

[48] Hu J-M, Duan C-G, Nan C-W and Chen L-Q 2017 Understanding and designing magnetoelectric heterostructures guided by computation: progresses, remaining questions, and perspectives $n p j$ Comput. Mater. 318

[49] Meisenheimer P B, Novakov S, Vu N M and Heron J T 2018 Perspective: Magnetoelectric switching in thin film multiferroic heterostructures J. Appl. Phys. 123240901

[50] Bibes M, Villegas J E and Barthélémy A 2011 Ultrathin oxide films and interfaces for electronics and spintronics Adv. Phys. 60 5-84

[51] Zhou J, Trassin M, He Q, Tamura N, Kunz M, Cheng C, Zhang J, Liang W-I, Seidel J, Hsin C-L and Wu J 2012 Directed assembly of nano-scale phase variants in highly strained BiFeO3 thin films J. Appl. Phys. 11264102

[52] Liu M, Li S, Zhou Z, Beguhn S, Lou J, Xu F, Jian Lu T and Sun N X 2012 Electrically induced enormous magnetic anisotropy in Terfenol-D/lead zinc niobate-lead titanate multiferroic heterostructures J. Appl. Phys. 11263917

[53] Liu M and Sun N X 2014 Voltage control of magnetism in multiferroic heterostructures Phil. Trans. R. Soc. A 37220120439

[54] Lahtinen T H E, Franke K J A and Van Dijken S 2012 Electric-field control of magnetic domain wall motion and local magnetization reversal Sci. Rep. 2258

[55] Franke K J A, López González D, Hämäläinen S J and van Dijken S 2014 Size Dependence of Domain Pattern Transfer in Multiferroic Heterostructures Phys. Rev. Lett. 11217201

[56] Buzzi M, Chopdekar R V, Hockel J L, Bur A, Wu T, Pilet N, Warnicke P, Carman G P, Heyderman L J and Nolting F 2013 Single Domain Spin Manipulation by Electric Fields in Strain Coupled Artificial Multiferroic Nanostructures Phys. Rev. Lett. 11127204 
[57] Lou J, Reed D, Pettiford C, Liu M, Han P, Dong S and Sun N X 2008 Giant microwave tunability in FeGaB/lead magnesium niobate-lead titanate multiferroic composites Appl. Phys. Lett. 92262502

[58] Callen E R and Callen H B 1963 Static Magnetoelastic Coupling in Cubic Crystals Phys. Rev. 129 578-93

[59] Newnham R E 2004 Properties of Materials: Anisotropy, Symmetry, Structure (OUP Oxford)

[60] Wu R 2002 Origin of large magnetostriction in FeGa alloys J. Appl. Phys. 91 7358-60

[61] Du Y, Huang M, Chang S, Schlagel D L, Lograsso T A and McQueeney R J 2010 Relation between Ga ordering and magnetostriction of Fe-Ga alloys studied by x-ray diffuse scattering Phys. Rev. $B$ 8154432

[62] Wang H, Zhang Y N, Wu R Q, Sun L Z, Xu D S and Zhang Z D 2013 Understanding strong magnetostriction in Fe 100-x Ga x alloys Sci. Rep. 3 1-5

[63] Furthmuller J, Fahnle M and Herzer G 1986 Theory of magnetostriction in amorphous and polycrystalline ferromagnets J. Phys. F Met. Phys. 16 L255--L258

[64] Wang Q, Li X, Liang C-Y, Barra A, Domann J, Lynch C, Sepulveda A and Carman G 2017 Strainmediated $180^{\circ}$ switching in CoFeB and Terfenol-D nanodots with perpendicular magnetic anisotropy Appl. Phys. Lett. 110102903

[65] Parkes D E, Cavill S A, Hindmarch A T, Wadley P, McGee F, Staddon C R, Edmonds K W, Campion R P, Gallagher B L and Rushforth A W 2012 Non-volatile voltage control of magnetization and magnetic domain walls in magnetostrictive epitaxial thin films Appl. Phys. Lett. 10172402

[66] Ahmad H, Atulasimha J and Bandyopadhyay S 2015 Reversible strain-induced magnetization switching in FeGa nanomagnets: Pathway to a rewritable, non-volatile, non-toggle, extremely low energy straintronic memory Sci. Rep. 5 srep18264

[67] Lee Y, Liu Z Q, Heron J T, Clarkson J D, Hong J, Ko C, Biegalski M D, Aschauer U, Hsu S L, Nowakowski M E, Wu J, Christen H M, Salahuddin S, Bokor J B, Spaldin N A, Schlom D G and Ramesh R 2015 Large resistivity modulation in mixed-phase metallic systems Nat. Commun. 6 ncomms6959

[68] Zhang S, Zhao Y, Xiao X, Wu Y, Rizwan S, Yang L, Li P, Wang J, Zhu M, Zhang H, Jin X and Han X 
2014 Giant electrical modulation of magnetization in Co40Fe40B20/Pb(Mg1/3Nb2/3)0.7Ti0.303(011) heterostructure Sci. Rep. 43727

[69] Feng M, Wang J, Hu J-M, Wang J, Ma J, Li H-B, Shen Y, Lin Y-H, Chen L-Q and Nan C-W 2015 Optimizing direct magnetoelectric coupling in $\mathrm{Pb}(\mathrm{Zr}, \mathrm{Ti}) \mathrm{O} 3 / \mathrm{Ni}$ multiferroic film heterostructures Appl. Phys. Lett. 10672901

[70] Lahtinen T H E, Tuomi J O and Van Dijken S 2011 Pattern transfer and electric-field-induced magnetic domain formation in multiferroic heterostructures Adv. Mater. 23 3187-91

[71] Lahtinen T H E and van Dijken S 2013 Temperature control of local magnetic anisotropy in multiferroic CoFe/BaTiO3 Appl. Phys. Lett. 102112406

[72] Kim J Y, Yao L and Van Dijken S 2013 Coherent piezoelectric strain transfer to thick epitaxial ferromagnetic films with large lattice mismatch J. Phys. Condens. Matter 25

[73] Franke K J A, Van de Wiele B, Shirahata Y, Hämälainen S J, Taniyama T and van Dijken S 2015 Reversible electric-field-driven magnetic domain-wall motion Phys. Rev. X 5011010

[74] Nikonov D E and Young I A 2015 Benchmarking of Beyond-CMOS Exploratory Devices for Logic Integrated Circuits IEEE J. Explor. Solid-State Comput. Devices Circuits 1 3-11

[75] Jaiswal A and Roy K 2017 MESL: Proposal for a Non-volatile Cascadable Magneto-Electric Spin Logic Sci. Rep. 739793

[76] Manipatruni S, Nikonov D E, Lin C-C, Gosavi T A, Liu H, Prasad B, Huang Y-L, Bonturim E, Ramesh R and Young I A 2019 Scalable energy-efficient magnetoelectric spin-orbit logic Nature 565 35-42

[77] Ma J, Shi Z and Nan C-W 2007 Magnetoelectric Properties of Composites of Single Pb(Zr,Ti)O3 Rods and Terfenol-D/Epoxy with a Single-Period of 1-3-Type Structure Adv. Mater. 19 2571-3

[78] Nan C-W, Liu G and Lin Y 2003 Influence of interfacial bonding on giant magnetoelectric response of multiferroic laminated composites of Tb1-xDyxFe2 and PbZrxTi1-xO3 Appl. Phys. Lett. 83 4366-8

[79] Vaz C A F, Hoffman J, Segal Y, Reiner J W, Grober R D, Zhang Z, Ahn C H and Walker F J 2010 Origin of the magnetoelectric coupling effect in $\mathrm{Pb}(\mathrm{ZrO} .2 \mathrm{Ti0.8}) \mathrm{O} 3$ / $\mathrm{La0} .8 \mathrm{SrO}$. 2MnO3 multiferroic heterostructures Phys. Rev. Lett. 104127202 
[80] Evans D M, Schilling A, Kumar A, Sanchez D, Ortega N, Arredondo M, Katiyar R S, Gregg J M and Scott J F 2013 Magnetic switching of ferroelectric domains at room temperature in multiferroic PZTFT Nat. Commun. 4:1534

[81] Zhang S, Zhao Y G, Li P S, Yang J J, Rizwan S, Zhang J X, Seidel J, Qu T L, Yang Y J, Luo Z L, He Q, Zou T, Chen Q P, Wang J W, Yang L F, Sun Y, Wu Y Z, Xiao X, Jin X F, Huang J, Gao C, Han X F and Ramesh R 2012 Electric-Field Control of Nonvolatile Magnetization in Co40Fe40B20/Pb(Mg1/3Nb2/3)0.7Ti0.3O3 Structure at Room Temperature Phys. Rev. Lett. 108 137203

[82] Wu T, Bur A, Wong K, Leon Hockel J, Hsu C-J, Kim H K D, Wang K L and Carman G P 2011 Electricpoling-induced magnetic anisotropy and electric-field-inducedmagnetization reorientation in magnetoelectric Ni/(011)[Pb(Mg1/3Nb2/3)O3](1-x)-[PbTiO3]x heterostructure J. Appl. Phys. 109 $07 D 732$

[83] Mardana A, Ducharme S and Adenwalla S 2011 Ferroelectric Control of Magnetic Anisotropy Nano Lett. $113862-7$

[84] Ghidini M, Pellicelli R, Prieto J L, Moya X, Soussi J, Briscoe J, Dunn S and Mathur N D 2013 Nonvolatile electrically-driven repeatable magnetization reversal with no applied magnetic field Nat. Commun. 41453

[85] Kittmann A, Durdaut P, Zabel S, Reermann J, Schmalz J, Spetzler B, Meyners D, Sun N X, McCord J, Gerken M, Schmidt G, Höft M, Knöchel R, Faupel F and Quandt E 2018 Wide Band Low Noise Love Wave Magnetic Field Sensor System Sci. Rep. 8278

[86] Lin H, Page M R, McConney M, Jones J, Howe B and Sun N X 2018 Integrated magnetoelectric devices: Filters, pico-Tesla magnetometers, and ultracompact acoustic antennas MRS Bull. $\mathbf{4 3}$ $841-7$

[87] Nan T, Lin H, Gao Y, Matyushov A, Yu G, Chen H, Sun N, Wei S, Wang Z, Li M, Wang X, Belkessam A, Guo R, Chen B, Zhou J, Qian Z, Hui Y, Rinaldi M, McConney M E, Howe B M, Hu Z, Jones J G, Brown G J and Sun N X 2017 Acoustically actuated ultra-compact NEMS magnetoelectric antennas Nat. Commun. 8296

[88] Lou J, Liu M, Reed D, Ren Y and Sun N X 2009 Giant Electric Field Tuning of Magnetism in Novel Multiferroic FeGaB/Lead Zinc Niobate-Lead Titanate (PZN-PT) Heterostructures Adv. Mater. 21 
4711-5

[89] Liu M, Obi O, Lou J, Chen Y, Cai Z, Stoute S, Espanol M, Lew M, Situ X, Ziemer K S, Harris V G and Sun N X 2009 Giant Electric Field Tuning of Magnetism in Novel Multiferroic FeGaB/Lead Zinc Niobate-Lead Titanate (PZN-PT) Heterostructures Adv. Funct. Mater. 19 1826-31

[90] Zaeimbashi M, Lin H, Dong C, Liang X, Nasrollahpour M, Chen H, Sun N, Matyushov A, He Y, Wang X, Tu C, Wei Y, Zhang Y, Cash S S, Onabajo M, Shrivastava A and Sun N 2019 NanoNeuroRFID: A Wireless Implantable Device Based on Magnetoelectric Antennas IEEE J. Electromagn. RF Microwaves Med. Biol. 3 206-15

[91] Emam S, Sun N-X and Nasrollahpour M 2019 P3-226: electrochemical gas sensor arrays for detecting volatile organic compound biomarkers in the exhaled breath Alzheimer's Dement. 15 P1018-P1018

[92] Jahjah W, Jay J-P, Le Grand Y, Fessant A, Prinsloo A R E, Sheppard C J, Dekadjevi D T and Spenato D 2020 Electrical Manipulation of Magnetic Anisotropy in a Fe81Ga19/Pb(Mg1/3Nb2/3)O3Pb(ZrxTi1-x)O3 Magnetoelectric Multiferroic Composite Phys. Rev. Appl. 1334015

[93] Wang J-L, Echtenkamp W, Mahmood A and Binek C 2019 Voltage controlled magnetism in Cr2O3 based all-thin-film systems J. Magn. Magn. Mater. 486165262

[94] Béa H, Bibes M, Ott F, Dupé B, Zhu X-H, Petit S, Fusil S, Deranlot C, Bouzehouane K and Barthélémy A 2008 Mechanisms of Exchange Bias with Multiferroic BiFeO3 Epitaxial Thin Films Phys. Rev. Lett. 10017204

[95] Trassin M, Luca G De, Manz S and Fiebig M 2015 Probing Ferroelectric Domain Engineering in BiFeO 3 Thin Films by Second Harmonic Generation 4871-6

[96] Gao T, Zhang X, Ratcliff W, Maruyama S, Murakami M, Varatharajan A, Yamani Z, Chen P, Wang K, Zhang H, Shull R, Bendersky L A, Unguris J, Ramesh R and Takeuchi I 2017 Electric-Field Induced Reversible Switching of the Magnetic Easy Axis in Co/BiFeO3 on SrTiO3 Nano Lett. 17 2825-32

[97] Heron J T, Bosse J L, He Q, Gao Y, Trassin M, Ye L, Clarkson J D, Wang C, Liu J, Salahuddin S, Ralph D C, Schlom D G, Íñiguez J, Huey B D and Ramesh R 2014 Deterministic switching of ferromagnetism at room temperature using an electric field Nature 516 370-3

[98] Trassin M, Clarkson J D, Bowden S R, Liu J, Heron J T, Paull R J, Arenholz E, Pierce D T and Unguris 
J 2013 Interfacial coupling in multiferroic/ferromagnet heterostructures Phys. Rev. B - Condens. Matter Mater. Phys. 87134426

[99] Radu F and Zabel H 2007 Exchange bias effect of ferro-/antiferromagnetic heterostructures Springer Tracts Mod. Phys. 227 97-184

[100] Toyoki K, Shiratsuchi Y, Kobane A, Mitsumata C, Kotani Y, Nakamura T and Nakatani R 2015 Magnetoelectric switching of perpendicular exchange bias in $\mathrm{Pt} / \mathrm{Co} / \alpha-\mathrm{Cr} 2 \mathrm{O} 3 / \mathrm{Pt}$ stacked films Appl. Phys. Lett. 106162404

[101] Borisov P, Hochstrat A, Chen X, Kleemann W and Binek C 2005 Magnetoelectric Switching of Exchange Bias Phys. Rev. Lett. 94117203

[102] Heron J T, Trassin M, Ashraf K, Gajek M, He Q, Yang S Y, Nikonov D E, Chu Y H, Salahuddin S and Ramesh R 2011 Electric-field-induced magnetization reversal in a ferromagnet-multiferroic heterostructure Phys. Rev. Lett. 107 1-5

[103] Wu S M, Cybart S A, Yu P, Rossell M D, Zhang J X, Ramesh R and Dynes R C 2010 Reversible electric control of exchange bias in a multiferroic field-effect device Nat. Mater. 9 756-61

[104] Wu S M, Cybart S A, Yi D, Parker J M, Ramesh R and Dynes R C 2013 Full Electric Control of Exchange Bias Phys. Rev. Lett. 11067202

[105] He X, Wang Y, Wu N, Caruso A N, Vescovo E, Belashchenko K D, Dowben P A and Binek C 2010 Robust isothermal electric control of exchange bias at room temperature Nat. Mater. 9 579-85

[106] Nozaki T, Al-Mahdawi M, Pati S P, Ye S, Shiokawa Y and Sahashi M 2017 Magnetoelectric switching energy in Cr2O3/Pt/Co perpendicular exchange coupled thin film system with small Cr2O3 magnetization Jpn. J. Appl. Phys. 5670302

[107] Kosub T, Kopte M, Hühne R, Appel P, Shields B, Maletinsky P, Hübner R, Liedke M O, Fassbender J, Schmidt O G and Makarov D 2017 Purely antiferromagnetic magnetoelectric random access memory Nat. Commun. 813985

[108] Borisov P, Ashida T, Nozaki T, Sahashi M and Lederman D 2016 Magnetoelectric properties of 500-nm Cr2O3 films Phys. Rev. B 93174415

[109] Vu N M, Luo X, Novakov S, Jin W, Nordlander J, Meisenheimer P B, Trassin M, Zhao L and Heron J 
T 2020 Bulk-like dielectric and magnetic properties of sub $100 \mathrm{~nm}$ thick single crystal Cr2O3 films on an epitaxial oxide electrode Sci. Rep. 1014721

[110] Gross I, Akhtar W, Garcia V, Martínez L J, Chouaieb S, Garcia K, Carrétéro C, Barthélémy A, Appel P, Maletinsky P, Kim J V., Chauleau J Y, Jaouen N, Viret M, Bibes M, Fusil S and Jacques V 2017 Real-space imaging of non-collinear antiferromagnetic order with a single-spin magnetometer Nature 549 252-6

[111] Sando D, Agbelele A, Rahmedov D, Liu J, Rovillain P, Toulouse C, Infante I C, Pyatakov A P, Fusil S, Jacquet E, Carrétéro C, Deranlot C, Lisenkov S, Wang D, Le Breton J-M, Cazayous M, Sacuto A, Juraszek J, Zvezdin A K, Bellaiche L, Dkhil B, Barthélémy A and Bibes M 2013 Crafting the magnonic and spintronic response of BiFeO3 films by epitaxial strain Nat. Mater. 12 641-6

[112] Martin L W, Chu Y H, Holcomb M B, Huijben M, Yu P, Han S J, Lee D, Wang S X and Ramesh R 2008 Nanoscale control of exchange bias with BiFeO 3 thin films Nano Lett. 8 2050-5

[113] Huang Y-L, Nikonov D, Addiego C, Chopdekar R V, Prasad B, Zhang L, Chatterjee J, Liu H-J, Farhan A, Chu Y-H, Yang M, Ramesh M, Qiu Z Q, Huey B D, Lin C-C, Gosavi T, Íñiguez J, Bokor J, Pan X, Young I, Martin L W and Ramesh R 2020 Manipulating magnetoelectric energy landscape in multiferroics Nat. Commun. 112836

[114] Wang J J, Hu J M, Ma J, Zhang J X, Chen L Q and Nan C W 2014 Full $180^{\circ}$ Magnetization Reversal with Electric Fields Sci. Rep. 47507

[115] Hu J-M, Yang T, Wang J, Huang H, Zhang J, Chen L-Q and Nan C-W 2015 Purely Electric-FieldDriven Perpendicular Magnetization Reversal Nano Lett. 15 616-22

[116] Imamura H, Nozaki T, Yuasa S and Suzuki Y 2018 Deterministic Magnetization Switching by Voltage Control of Magnetic Anisotropy and Dzyaloshinskii-Moriya Interaction under an In-Plane Magnetic Field Phys. Rev. Appl. 1054039

[117] Zavaliche F, Zhao T, Zheng H, Straub F, Cruz M P, Yang P-L, Hao D and Ramesh R 2007 Electrically Assisted Magnetic Recording in Multiferroic Nanostructures Nano Lett. 7 1586-90

[118] Skumryev V, Laukhin V, Fina I, Martí X, Sánchez F, Gospodinov M and Fontcuberta J 2011 Magnetization reversal by electric-field decoupling of magnetic and ferroelectric domain walls in multiferroic-based heterostructures Phys. Rev. Lett. 106057206 
[119] Kani N, Heron J T and Naeemi A 2017 Strain-Mediated Magnetization Reversal Through SpinTransfer Torque IEEE Trans. Magn. PP 1

[120] Roy K, Bandyopadhyay S and Atulasimha J 2011 Hybrid spintronics and straintronics: A magnetic technology for ultra low energy computing and signal processing Appl. Phys. Lett. 9963108

[121] Morelli A, Johann F, Burns S R, Douglas A and Gregg J M 2016 Deterministic Switching in Bismuth Ferrite Nanoislands Nano Lett. 16 5228-34

[122] Prasad B, Huang Y-L, Chopdekar R V, Chen Z, Steffes J, Das S, Li Q, Yang M, Lin C-C, Gosavi T, Nikonov D E, Qiu Z Q, Martin L W, Huey B D, Young I, íñiguez J, Manipatruni S and Ramesh R 2020 Ultralow Voltage Manipulation of Ferromagnetism Adv. Mater. 322001943

[123] Parsonnet E, Huang Y-L, Gosavi T, Qualls A, Nikonov D, Lin C-C, Young I, Bokor J, Martin L W and Ramesh R 2020 Toward Intrinsic Ferroelectric Switching in Multiferroic BiFeO3 Phys. Rev. Lett. 12567601

[124] Nagarajan V, Ganpule C S, Li H, Salamanca-Riba L, Roytburd A L, Williams E D and Ramesh R 2001 Control of domain structure of epitaxial PbZr0.2Ti0.803 thin films grown on vicinal (001) SrTiO3 substrates Appl. Phys. Lett. 79 2805-7

[125] Chu Y H, Cruz M P, Yang C H, Martin L W, Yang P L, Zhang J X, Lee K, Yu P, Chen L Q and Ramesh R 2007 Domain control in multiferroic BiFeO3 through substrate vicinality Adv. Mater. 19 2662-6

[126] Xu R, Liu S, Grinberg I, Karthik J, Damodaran A R, Rappe A M and Martin L W 2015 Ferroelectric polarization reversal via successive ferroelastic transitions Nat. Mater. 14 79-86

[127] Chu Y H, He Q, Yang C H, Yu P, Martin L W, Shafer P and Ramesh R 2009 Nanoscale control of domain architectures in BiFeO 3 thin films Nano Lett. 9 1726-30

[128] FeigI L, Yudin P, Stolichnov I, Sluka T, Shapovalov K, Mtebwa M, Sandu C S, Wei X K, Tagantsev A K and Setter N 2014 Controlled stripes of ultrafine ferroelectric domains Nat. Commun. 54677

[129] Haeni J H, Irvin P, Chang W, Uecker R, Reiche P, Li Y L, Choudhury S, Tian W, Hawley M E, Craigo B, Tagantsev A K, Pan X Q, Streiffer S K, Chen L Q, Kirchoefer S W, Levy J and Schlom D G 2004 Room-temperature ferroelectricity in strained SrTiO3 Nature 430 758-61

[130] Lee J H, Fang L, Vlahos E, Ke X, Jung Y W, Kourkoutis L F, Kim J W, Ryan P J, Heeg T, Roeckerath M, 
Goian V, Bernhagen M, Uecker R, Hammel P C, Rabe K M, Kamba S, Schubert J, Freeland J W, Muller D A, Fennie C J, Schiffer P, Gopalan V, Johnston-Halperin E and Schlom D G 2010 A strong ferroelectric ferromagnet created by means of spin-lattice coupling Nature 466 954-8

[131] Becher C, Maurel L, Aschauer U, Lilienblum M, Magén C, Meier D, Langenberg E, Trassin M, Blasco J, Krug I P, Algarabel P A, Spaldin N A, Pardo J A and Fiebig M 2015 Strain-induced coupling of electrical polarization and structural defects in SrMnO 3 films Nat. Nanotechnol. 10 661-5

[132] Guo J W, Wang P S, Yuan Y, He Q, Lu J L, Chen T Z, Yang S Z, Wang Y J, Erni R, Rossell M D, Gopalan V, Xiang H J, Tokura Y and Yu P 2018 Strain-induced ferroelectricity and spin-lattice coupling in SrMnO3 thin films Phys. Rev. B 97235135

[133] Zhang Y, Wang J, Sahoo M P K, Shimada T and Kitamura T 2017 Strain-induced ferroelectricity and lattice coupling in $\mathrm{BaSnO} 3$ and SrSnO3 Phys. Chem. Chem. Phys. 19 26047-55

[134] Sando D, Han M J, Govinden V, Paull O, Appert F, Carrétéro C, Fischer J, Barthélémy A, Bibes M, Garcia V, Fusil S, Dkhil B, Juraszek J, Zhu Y, Ma X L and Nagarajan V 2020 Interfacial Strain Gradients Control Nanoscale Domain Morphology in Epitaxial BiFeO3 Multiferroic Films Adv. Func. Mat. 2000343

[135] Agbelele A, Sando D, Toulouse C, Paillard C, Johnson R D, Rüffer R, Popkov A F, Carrétéro C, Rovillain P, Le Breton J M, Dkhil B, Cazayous M, Gallais Y, Méasson M A, Sacuto A, Manuel P, Zvezdin A K, Barthélémy A, Juraszek J and Bibes M 2017 Strain and Magnetic Field Induced SpinStructure Transitions in Multiferroic BiFeO3 Adv. Mater. 291602327

[136] Sando D, Appert F, Xu B, Paull O, Burns S R, Carrétéro C, Dupé B, Garcia V, Gallais Y, Sacuto A, Cazayous M, Dkhil B, Le Breton J M, Barthélémy A, Bibes M, Bellaiche L, Nagarajan V and Juraszek J 2019 A magnetic phase diagram for nanoscale epitaxial BiFeO3 films Appl. Phys. Rev. 6041404

[137] Chen Z, Chen Z, Kuo C Y, Tang Y, Dedon L R, Li Q, Zhang L, Klewe C, Huang Y L, Prasad B, Farhan A, Yang M, Clarkson J D, Das S, Manipatruni S, Tanaka A, Shafer P, Arenholz E, Scholl A, Chu Y H, Qiu Z Q, Hu Z, Tjeng L H, Ramesh R, Wang L W and Martin L W 2018 Complex strain evolution of polar and magnetic order in multiferroic BiFeO3 thin films Nat. Commun. 93764

[138] Waterfield Price N, Vibhakar A M, Johnson R D, Schad J, Saenrang W, Bombardi A, Chmiel F P, Eom C B and Radaelli P G 2019 Strain Engineering a Multiferroic Monodomain in Thin-Film BiFe O3 Phys. Rev. Appl. 11024035 
[139] Saenrang W, Davidson B A, Maccherozzi F, Podkaminer J P, Irwin J, Johnson R D, Freeland J W, Íñiguez J, Schad J L, Reierson K, Frederick J C, Vaz C A F, Howald L, Kim T H, Ryu S, Veenendaal M V., Radaelli P G, Dhesi S S, Rzchowski M S and Eom C B 2017 Deterministic and robust roomtemperature exchange coupling in monodomain multiferroic $\mathrm{BiFeO} 3$ heterostructures Nat. Commun. 81583

[140] Fong D D, Stephenson G B, Streiffer S K, Eastman J A, Aucielo O, Fuoss P H and Thompson C 2004 Ferroelectricity in ultrathin perovskite films Science 304 1650-3

[141] De Luca G, Strkalj N, Manz S, Bouillet C, Fiebig M and Trassin M 2017 Nanoscale design of polarization in ultrathin ferroelectric heterostructures Nat. Commun. 81419

[142] Choi K J, Biegalski M, Li Y L, Sharan A, Schubert J, Uecker R, Reiche P, Chen Y B, Pan X Q, Gopalan V, Chen L-Q, Schlom D G and Eom C B 2004 Enhancement of Ferroelectricity in Strained BaTiO3 Thin Films Science 306 1005-9

[143] Liu G, Chen J, Lichtensteiger C, Triscone J M, Aguado-Puente P, Junquera J and Valanoor N 2016 Positive Effect of an Internal Depolarization Field in Ultrathin Epitaxial Ferroelectric Films Adv. Electron. Mater. 21500288

[144] Lichtensteiger C, Fernandez-Pena S, Weymann C, Zubko P and Triscone J M 2014 Tuning of the depolarization field and nanodomain structure in ferroelectric thin films Nano Lett. 14 4205-11

[145] Liu Y, Lou X, Bibes M and Dkhil B 2013 Effect of a built-in electric field in asymmetric ferroelectric tunnel junctions Phys. Rev. B - Condens. Matter Mater. Phys. 88 1-8

[146] Simmons J G 1963 Intrinsic fields in thin insulating films between dissimilar electrodes Phys. Rev. Lett. $1010-2$

[147] Lichtensteiger C, Weymann C, Fernandez-Pena S, Paruch P and Triscone J M 2016 Built-in voltage in thin ferroelectric PbTiO3 films: The effect of electrostatic boundary conditions New J. Phys. 18 043030

[148] Balke N, Ramesh R and Yu P 2017 Manipulating Ferroelectrics through Changes in Surface and Interface Properties ACS Appl. Mater. Interfaces 9 39736-46

[149] Chen D, Chen Z, He Q, Clarkson J D, Serrao C R, Yadav A K, Nowakowski M E, Fan Z, You L, Gao X, Zeng D, Chen L, Borisevich A Y, Salahuddin S, Liu J M and Bokor J 2017 Interface Engineering of 
Domain Structures in BiFeO3 Thin Films Nano Lett. 17 486-93

[150] Seidel J, Martin L W, He Q, Zhan Q, Chu Y H, Rother A, Hawkridge M E, Maksymovych P, Yu P, Gajek M, Balke N, Kalinin S V., Gemming S, Wang F, Catalan G, Scott J F, Spaldin N A, Orenstein J and Ramesh R 2009 Conduction at domain walls in oxide multiferroics Nat. Mater. 8 229-34

[151] Campanini M, Gradauskaite E, Trassin M, Yi D, Yu P, Ramesh R, Erni R and Rossell M D 2020 Imaging and quantification of charged domain walls in BiFeO3 Nanoscale 12 9186-93

[152] Yu P, Luo W, Yi D, Zhang J X, Rossell M D, Yang C-H, You L, Singh-Bhalla G, Yang S Y, He Q, Ramasse Q M, Erni R, Martin L W, Chu Y H, Pantelides S T, Pennycook S J and Ramesh R 2012 Interface control of bulk ferroelectric polarization Proc. Natl. Acad. Sci. 109 9710-5

[153] Yi D, Yu P, Chen Y C, Lee H H, He Q, Chu Y H and Ramesh R 2019 Tailoring Magnetoelectric Coupling in $\mathrm{BiFeO} / \mathrm{La0} .7 \mathrm{Sr} 0.3 \mathrm{MnO} 3$ Heterostructure through the Interface Engineering $\mathrm{Adv}$. Mater. 1806335

[154] Li L, Jokisaari J R, Zhang Y, Cheng X, Yan X, Heikes C, Lin Q, Gadre C, Schlom D G, Chen L Q and Pan X 2018 Control of Domain Structures in Multiferroic Thin Films through Defect Engineering Adv. Mater. 301802737

[155] Puggioni D, Giovannetti G and Rondinelli J M 2018 Polar metals as electrodes to suppress the critical-thickness limit in ferroelectric nanocapacitors J. Appl. Phys. 124174102

[156] Tian Y, Wei L, Zhang Q, Huang H, Zhang Y, Zhou H, Ma F, Gu L, Meng S, Chen L Q, Nan C W and Zhang J 2018 Water printing of ferroelectric polarization Nat. Commun. 93809

[157] Chopdekar R V., Malik V K, Fraile Rodríguez A, Le Guyader L, Takamura Y, Scholl A, Stender D, Schneider C W, Bernhard C, Nolting F and Heyderman L J 2012 Spatially resolved strain-imprinted magnetic states in an artificial multiferroic Phys. Rev. B - Condens. Matter Mater. Phys. 86014408

[158] Lahtinen T H E, Franke K J A and Dijken S van 2012 Electric-field control of magnetic domain wall motion and local magnetization reversal Sci. Rep. 2258

[159] You L, Wang B, Zou X, Lim Z S, Zhou Y, Ding H, Chen L and Wang J 2013 Origin of the uniaxial magnetic anisotropy in La0.7Sr 0.3MnO3 on stripe-domain BiFeO3 Phys. Rev. B - Condens. Matter Mater. Phys. 88 1-9 
[160] Streubel R, Köhler D, Schäfer R and Eng L M 2013 Strain-mediated elastic coupling in magnetoelectric nickel/barium-titanate heterostructures Phys. Rev. B - Condens. Matter Mater. Phys. 87054410

[161] Brandl F, Franke K J A, Lahtinen T H E, Van Dijken S and Grundler D 2014 Spin waves in CoFeB on ferroelectric domains combining spin mechanics and magnonics Solid State Commun. 198 13-7

[162] Fackler S W, Donahue M J, Gao T, Nero P N A, Cheong S W, Cumings J and Takeuchi I 2014 Local control of magnetic anisotropy in transcritical permalloy thin films using ferroelectric BaTiO3 domains Appl. Phys. Lett. 105212905

[163] Wang J, Pesquera D, Mansell R, Van Dijken S, Cowburn R P, Ghidini M and Mathur N D 2019 Giant non-volatile magnetoelectric effects via growth anisotropy in Co40Fe40B20 films on PMN-PT substrates Appl. Phys. Lett. 114092401

[164] Phillips L C, Cherifi R O, Ivanovskaya V, Zobelli A, Infante I C, Jacquet E, Guiblin N, Ünal A A, Kronast F, Dkhil B, Barthélémy A, Bibes M and Valencia S 2015 Local electrical control of magnetic order and orientation by ferroelastic domain arrangements just above room temperature Sci. Rep. 510026

[165] Franke K J A, Lahtinen T H E and Van Dijken S 2012 Field tuning of ferromagnetic domain walls on elastically coupled ferroelectric domain boundaries Phys. Rev. B - Condens. Matter Mater. Phys. 85094423

[166] Kim S H, Choi H, No K and Shin S C 2010 Electric-field induced ferromagnetic domain changes in exchange biased Co-BiFeO3 composites J. Phys. D. Appl. Phys. 43165001

[167] Cybart S A, Rossell M D, Wu S M, Zhang J X, Yu P, Ramesh R and Dynes R C 2010 Reversible electric control of exchange bias in a multiferroic field-effect device Nat. Mater. 9 756-61

[168] Laukhin V, Skumryev V, Martí X, Hrabovsky D, Sánchez F, García-Cuenca M V., Ferrater C, Varela M, Lüders U, Bobo J F and Fontcuberta J 2006 Electric-field control of exchange bias in multiferroic epitaxial heterostructures Phys. Rev. Lett. 97227201

[169] Duan C G, Jaswal S S and Tsymbal E Y 2006 Predicted magnetoelectric effect in Fe/BaTiO3 multilayers: Ferroelectric control of magnetism Phys. Rev. Lett. 97047201

[170] Rondinelli J M, Stengel M and Spaldin N A 2008 Carrier-mediated magnetoelectricity in complex 
oxide heterostructures Nat. Nanotechnol. 3 46-50

[171] Molegraaf H J A, Hoffman J, Vaz C A F, Gariglio S, van der Marel D, Ahn C H and Triscone J-M 2009 Magnetoelectric Effects in Complex Oxides with Competing Ground States Adv. Mater. 21 3470-4

[172] Cai T, Ju S, Lee J, Sai N, Demkov A A, Niu Q, Li Z, Shi J and Wang E 2009 Magnetoelectric coupling and electric control of magnetization in ferromagnet/ferroelectric/normal-metal superlattices Phys. Rev. B - Condens. Matter Mater. Phys. 80 140415(R)

[173] Brivio S, Cantoni M, Petti D and Bertacco R 2010 Near-room-temperature control of magnetization in field effect devices based on La0.67Sr0.33MnO3 thin films J. Appl. Phys. 108 113906

[174] Spurgeon S R, Balachandran P V., Kepaptsoglou D M, Damodaran A R, Karthik J, Nejati S, Jones L, Ambaye H, Lauter V, Ramasse Q M, Lau K K S, Martin L W, Rondinelli J M and Taheri M L 2015 Polarization screening-induced magnetic phase gradients at complex oxide interfaces Nat. Commun. 66735

[175] Avula S R V, Heidler J, Dreiser J, Vijayakumar J, Howald L, Nolting F and Piamonteze C 2018 Study of magneto-electric coupling between ultra-thin Fe films and PMN-PT using X-ray magnetic circular dichroism J. Appl. Phys. 123064103

[176] Hong X, Posadas A and Ahn C H 2005 Examining the screening limit of field effect devices via the metal-insulator transition Appl. Phys. Lett. 86142501

[177] De Luca G, Schoenherr P, Mendil J, Meier D, Fiebig M and Trassin M 2018 Domain-Pattern Transfer across an Artificial Magnetoelectric Interface Phys. Rev. Appl. 10054030

[178] Gao Y, Hu J M, Wu L and Nan C W 2015 Dynamic in situ visualization of voltage-driven magnetic domain evolution in multiferroic heterostructures J. Phys. Condens. Matter 27504005

[179] López González D, Shirahata Y, Van de Wiele B, Franke K J A, Casiraghi A, Taniyama T and van Dijken S 2017 Electric-field-driven domain wall dynamics in perpendicularly magnetized multilayers AIP Adv. 7035119

[180] Van De Wiele B, Leliaert J, Franke K J A and Van Dijken S 2016 Electric-field-driven dynamics of magnetic domain walls in magnetic nanowires patterned on ferroelectric domains New J. Phys. 18 033027 
[181] Ghidini M, Mansell R, Maccherozzi F, Moya X, Phillips L C, Yan W, Pesquera D, Barnes C H W, Cowburn R P, Hu J M, Dhesi S S and Mathur N D 2019 Shear-strain-mediated magnetoelectric effects revealed by imaging Nat. Mater. 18 840-5

[182] Leo N, Carolus V, White J S, Kenzelmann M, Hudl M, Tolédano P, Honda T, Kimura T, Ivanov S A, Weil M, Lottermoser T, Meier D and Fiebig M 2018 Magnetoelectric inversion of domain patterns Nature 560 466-70

[183] Hassanpour E, Weber M C, Bortis A, Tokunaga Y, Taguchi Y, Tokura Y, Cano A, Lottermoser T and Fiebig M 2019 Interconversion of multiferroic domains and domain walls arXiv: 1908.06876

[184] Meier D 2015 Functional domain walls in multiferroics J. Phys. Condens. Matter 27463003

[185] Gruverman A, Alexe M and Meier D 2019 Piezoresponse force microscopy and nanoferroic phenomena Nat. Commun. 101661

[186] Evans D M, Garcia V, Meier D and Bibes M 2020 Domains and domain walls in multiferroics Phys. Sci. Rev. 5

[187] Whyte J R, McQuaid R G P, Sharma P, Canalias C, Scott J F, Gruverman A and Gregg J M 2014 Ferroelectric domain wall injection Adv. Mater. 26 293-8

[188] McQuaid R G P, Campbell M P, Whatmore R W, Kumar A and Marty Gregg J 2017 Injection and controlled motion of conducting domain walls in improper ferroelectric $\mathrm{Cu}-\mathrm{Cl}$ boracite $\mathrm{Nat}$. Commun. 815105

[189] Sharma P, Sando D, Zhang Q, Cheng X, Prosandeev S, Bulanadi R, Prokhorenko S, Bellaiche L, Chen L Q, Nagarajan V and Seidel J 2019 Conformational Domain Wall Switch Adv. Funct. Mater. 291807523

[190] Whyte J R and Gregg J M 2015 A diode for ferroelectric domain-wall motion Nat. Commun. 6 7361

[191] McGilly L J, Yudin P, FeigI L, Tagantsev A K and Setter N 2015 Controlling domain wall motion in ferroelectric thin films Nat. Nanotechnol. 10 145-50

[192] Guo E J, Roth R, Herklotz A, Hesse D and Dörr K 2015 Ferroelectric $180^{\circ}$ domain wall motion controlled by biaxial strain Adv. Mater. 27 1615-8 
[193] De Luca G, Rossell M D, Schaab J, Viart N, Fiebig M and Trassin M 2017 Domain Wall Architecture in Tetragonal Ferroelectric Thin Films Adv. Mater. 291605145

[194] Cherifi-Hertel S, Bulou H, Hertel R, Taupier G, Dorkenoo K D H, Andreas C, Guyonnet J, Gaponenko I, Gallo K and Paruch P 2017 Non-Ising and chiral ferroelectric domain walls revealed by nonlinear optical microscopy Nat. Commun. 815768

[195] Nataf G F, Barrett N, Kreisel J and Guennou M 2018 Raman signatures of ferroic domain walls captured by principal component analysis J. Phys. Condens. Matter 30035902

[196] Hadjimichael M, Zatterin E, Fernandez-Peña S, Leake S J and Zubko P 2018 Domain Wall Orientations in Ferroelectric Superlattices Probed with Synchrotron X-Ray Diffraction Phys. Rev. Lett. 120037602

[197] Li D, Huang X, Xiao Z, Chen H, Zhang L, Hao Y, Song J, Shao D F, Tsymbal E Y, Lu Y and Hong X 2020 Polar coupling enabled nonlinear optical filtering at MoS2/ferroelectric heterointerfaces Nat. Commun. 111422

[198] Meier D, Maringer M, Lottermoser T, Becker P, Bohatý L and Fiebig M 2009 Observation and coupling of domains in a spin-spiral multiferroic Phys. Rev. Lett. 102107202

[199] Leo N, Bergman A, Cano A, Poudel N, Lorenz B, Fiebig M and Meier D 2015 Polarization control at spin-driven ferroelectric domain walls Nat. Commun. 66661

[200] Tokunaga Y, Furukawa N, Sakai H, Taguchi Y, Arima T H and Tokura Y 2009 Composite domain walls in a multiferroic perovskite ferrite Nat. Mater. 8 558-62

[201] Tokunaga Y, Taguchi Y, Arima T H and Tokura Y 2012 Electric-field-induced generation and reversal of ferromagnetic moment in ferrites Nat. Phys. 8 838-44

[202] Matsubara M, Manz S, Mochizuki M, Kubacka T, lyama A, Aliouane N, Kimura T, Johnson S L, Meier D and Fiebig M 2015 Magnetoelectric domain control in multiferroic TbMnO3 Science 348 $1112-5$

[203] Geng Y, Lee N, Choi Y J, Cheong S W and Wu W 2012 Collective magnetism at multiferroic vortex domain walls Nano Lett. 12 6055-9

[204] Xu T, Shimada T, Araki Y, Wang J and Kitamura T 2016 Multiferroic Domain Walls in Ferroelectric 
PbTiO3 with Oxygen Deficiency Nano Lett. 16 454-8

[205] Farokhipoor S, Magén C, Venkatesan S, Íñiguez J, Daumont C J M, Rubi D, Snoeck E, Mostovoy M, De Graaf C, Müller A, Döblinger M, Scheu C and Noheda B 2014 Artificial chemical and magnetic structure at the domain walls of an epitaxial oxide Nature 515 379-83

[206] Logginov A S, Meshkov G A, Nikolaev A V., Nikolaeva E P, Pyatakov A P and Zvezdin A K 2008 Room temperature magnetoelectric control of micromagnetic structure in iron garnet films Appl. Phys. Lett. 93182510

[207] Fontcuberta J, Skumryev V, Laukhin V, Granados X and Salje E K H 2015 Polar domain walls trigger magnetoelectric coupling Sci. Rep. 513784

[208] Salje E K H and Ding X 2016 Ferroelastic domain Boundary-Based multiferroicity Crystals 6163

[209] Nataf G F, Guennou M, Kreisel J, Hicher P, Haumont R, Aktas O, Salje E K H, Tortech L, Mathieu C, Martinotti D and Barrett N 2017 Control of surface potential at polar domain walls in a nonpolar oxide Phys. Rev. Mater. 1074410

[210] Wei X K, Sluka T, Fraygola B, Feigl L, Du H, Jin L, Jia C L and Setter N 2017 Controlled Charging of Ferroelastic Domain Walls in Oxide Ferroelectrics ACS Appl. Mater. Interfaces 9 6539-46

[211] Burton J D and Tsymbal E Y 2009 Prediction of electrically induced magnetic reconstruction at the manganite/ferroelectric interface Phys. Rev. B - Condens. Matter Mater. Phys. 80174406

[212] McNulty J A, Tran T T, Halasyamani P S, McCartan S J, MacLaren I, Gibbs A S, Lim F J Y, Turner P W, Gregg J M, Lightfoot P and Morrison F D 2019 An Electronically Driven Improper Ferroelectric: Tungsten Bronzes as Microstructural Analogs for the Hexagonal Manganites Adv. Mater. 31 1903620

[213] Moure A 2018 Review and Perspectives of Aurivillius Structures as a Lead-Free Piezoelectric System Appl. Sci. 862

[214] Birenbaum A Y and Ederer C 2014 Potentially multiferroic Aurivillius phase Bi5FeTi3 015: Cation site preference, electric polarization, and magnetic coupling from first principles Phys. Rev. B 90 214109

[215] Zhai X, Grutter A J, Yun Y, Cui Z and Lu Y 2018 Weak magnetism of Aurivillius-type multiferroic 
thin films probed by polarized neutron reflectivity Phys. Rev. Mater. 2044405

[216] Yang J, Zhu X, Wang F, Song D, Chen L and Yang B 2018 Evolution of structure and ferroelectricity in Aurivillius Bi 4 Bi n-3 Fe n-3 Ti 30 3n+3 thin films J. Mater. Chem. C 6 8618-27

[217] Djani H, Garcia-Castro A C, Tong W Y, Barone P, Bousquet E, Picozzi S and Ghosez P 2019 Rationalizing and engineering Rashba spin-splitting in ferroelectric oxides npj Quantum Mater. 4 51

[218] Campanini M, Trassin M, Ederer C, Erni R and Rossell M D 2019 Buried In-Plane Ferroelectric Domains in Fe-Doped Single-Crystalline Aurivillius Thin Films ACS Appl. Electron. Mater. 1 1019-28

[219] Zhu L, Ralph D C and Buhrman R A 2018 Highly Efficient Spin-Current Generation by the Spin Hall Effect in Au1-xPtx Phys. Rev. Appl. 1031001

[220] Zhu L, Zhu L, Shi S, Ralph D C and Buhrman R A 2020 Energy-Efficient Ultrafast SOT-MRAMs Based on Low-Resistivity Spin Hall Metal Au0.25Pt0.75 Adv. Electron. Mater. 6 1-7

[221] Binek C and Doudin B 2005 Magnetoelectronics with magnetoelectrics J. Phys. Condens. Matter 17 L39-44

[222] Manipatruni S, Nikonov D E, Lin C-C, Prasad B, Huang Y-L, Damodaran A R, Chen Z, Ramesh R and Young I A 2018 Voltage control of unidirectional anisotropy in ferromagnet-multiferroic system Sci. Adv. 4 eaat4229

[223] Heron J T 2013 Electric Field Control of Ferromagnetism and Magnetic Devices Using Multiferroics PhD Thesis, Univ. California, Berkeley

[224] Dowben P A, Binek C, Zhang K, Wang L, Mei W N, Bird J P, Singisetti U, Hong X, Wang K L and Nikonov D 2018 Towards a Strong Spin-Orbit Coupling Magnetoelectric Transistor IEEE J. Explor. Solid-State Comput. Devices Circuits 4 1-9

[225] Young I A 2018 IEEE Journal on Exploratory Solid-State Computational Devices and Circuits IEEE J. Explor. Solid-State Comput. Devices Circuits 4

[226] Pan C, Lou Q, Niemier M, Hu S and Naeemi A 2019 Energy-Efficient Convolutional Neural Network Based on Cellular Neural Network Using Beyond-CMOS Technologies IEEE J. Explor. SolidState Comput. Devices Circuits 5 85-93 
[227] Stephan A W, Hu J and Koester S J 2019 Performance Estimate of Inverse Rashba-Edelstein Magnetoelectric Devices for Neuromorphic Computing IEEE J. Explor. Solid-State Comput. Devices Circuits 5 25-33

[228] Lesne E, Fu Y, Oyarzun S, Rojas-Sánchez J C, Vaz D C, Naganuma H, Sicoli G, Attané J P, Jamet M, Jacquet E, George J M, Barthélémy A, Jaffrès H, Fert A, Bibes M and Vila L 2016 Highly efficient and tunable spin-to-charge conversion through Rashba coupling at oxide interfaces Nat. Mater. $151261-6$

[229] Yu G, Upadhyaya P, Fan Y, Alzate J G, Jiang W, Wong K L, Takei S, Bender S A, Chang L-T, Jiang Y, Lang M, Tang J, Wang Y, Tserkovnyak Y, Amiri P K and Wang K L 2014 Switching of perpendicular magnetization by spin-orbit torques in the absence of external magnetic fields Nat. Nanotechnol. $9548-54$

[230] Wang Z, Li Z, Wang M, Wu B, Zhu D and Zhao W 2019 Field-free spin-orbit-torque switching of perpendicular magnetization aided by uniaxial shape anisotropy Nanotechnology 30375202

[231] Lau Y-C, Betto D, Rode K, Coey J M D and Stamenov P 2016 Spin-orbit torque switching without an external field using interlayer exchange coupling Nat. Nanotechnol. 11 758-62

[232] Van Den Brink A, Vermijs G, Solignac A, Koo J, Kohlhepp J T, Swagten H J M and Koopmans B 2016 Field-free magnetization reversal by spin-Hall effect and exchange bias Nat. Commun. 7 1-6

[233] Cai K, Yang M, Ju H, Wang S, Ji Y, Li B, Edmonds K W, Sheng Y, Zhang B, Zhang N, Liu S, Zheng H and Wang K 2017 Electric field control of deterministic current-induced magnetization switching in a hybrid ferromagnetic/ferroelectric structure Nat. Mater. 16 712-6

[234] Nan T, Hu J-M, Dai M, Emori S, Wang X, Hu Z, Matyushov A, Chen L-Q and Sun N 2019 A StrainMediated Magnetoelectric-Spin-Torque Hybrid Structure Adv. Funct. Mater. 291806371

[235] Laanait N, Saenrang W, Zhou H, Eom C B and Zhang Z 2017 Dynamic X-ray diffraction imaging of the ferroelectric response in bismuth ferrite Adv. Struct. Chem. Imaging 311

[236] Liu L, Rojac T, Damjanovic D, Di Michiel M and Daniels J 2018 Frequency-dependent decoupling of domain-wall motion and lattice strain in bismuth ferrite Nat. Commun. 9 1-10

[237] Matveyev Y, Negrov D, Chernikova A, Lebedinskii Y, Kirtaev R, Zarubin S, Suvorova E, Gloskovskii A and Zenkevich A 2017 Effect of Polarization Reversal in Ferroelectric TiN/Hf0.5Zr0.5O2/TiN 
Devices on Electronic Conditions at Interfaces Studied in Operando by Hard X-ray Photoemission Spectroscopy ACS Appl. Mater. Interfaces 9 43370-6

[238] Nordlander J, De Luca G, Strkalj N, Fiebig M and Trassin M 2018 Probing ferroic states in oxide thin films using optical second harmonic generation Appl. Sci. 8570

[239] Gorfman S, Bokov A A, Davtyan A, Reiser M, Xie Y, Ye Z G, Zozulya A V, Sprung M, Pietsch U and Gutt C 2018 Ferroelectric domain wall dynamics characterized with X-ray photon correlation spectroscopy Proc. Natl. Acad. Sci. U. S. A. 115 E6680-9

[240] Agar J C, Naul B, Pandya S, van der Walt S, Maher J, Ren Y, Chen L Q, Kalinin S V, Vasudevan R K, Cao Y, Bloom J S and Martin L W 2019 Revealing ferroelectric switching character using deep recurrent neural networks Nat. Commun. 10 1-11

[241] Degen C L 2008 Scanning magnetic field microscope with a diamond single-spin sensor Appl. Phys. Lett. 92

[242] Maze J R, Stanwix P L, Hodges J S, Hong S, Taylor J M, Cappellaro P, Jiang L, Dutt M V G, Togan E, Zibrov A S, Yacoby A, Walsworth R L and Lukin M D 2008 Nanoscale magnetic sensing with an individual electronic spin in diamond Nature 455 644-7

[243] Rondin L, Tetienne J P, Hingant T, Roch J F, Maletinsky P and Jacques V 2014 Magnetometry with nitrogen-vacancy defects in diamond Reports Prog. Phys. 77

[244] Gross I, Akhtar W, Garcia V, Martínez L J, Chouaieb S, Garcia K, Carrétéro C, Barthélémy A, Appel P, Maletinsky P, Kim J-V, Chauleau J Y, Jaouen N, Viret M, Bibes M, Fusil S and Jacques V 2017 Real-space imaging of non-collinear antiferromagnetic order with a single-spin magnetometer Nature 549252

[245] Geng Y, Das H, Wysocki A L, Wang X, Cheong S-W, Mostovoy M, Fennie C J and Wu W 2014 Direct visualization of magnetoelectric domains Nat. Mater. 13 163-7

[246] Schoenherr P, Giraldo L, Lilienblum M, Trassin M, Meier D and Fiebig M 2017 Magnetoelectric Force Microscopy on Antiferromagnetic 180॰ Domains in Cr2O3 Materials (Basel). 101051

[247] Denev S A, Lummen T T A, Barnes E, Kumar A and Gopalan V 2011 Probing ferroelectrics using optical second harmonic generation J. Am. Ceram. Soc. 94 2699-727 
[248] Fiebig M, Pavlov V V. and Pisarev R V. 2005 Second-harmonic generation as a tool for studying electronic and magnetic structures of crystals: review J. Opt. Soc. Am. B 2296

[249] Tzschaschel C, Satoh T and Fiebig M 2019 Tracking the ultrafast motion of an antiferromagnetic order parameter Nat. Commun. 10 1-6

[250] Chauleau J Y, Haltz E, Carrétéro C, Fusil S and Viret M 2017 Multi-stimuli manipulation of antiferromagnetic domains assessed by second-harmonic imaging Nat. Mater. 16 803-7

[251] Stanciu C D, Hansteen F, Kimel A V., Kirilyuk A, Tsukamoto A, Itoh A and Rasing T 2007 All-optical magnetic recording with circularly polarized light Phys. Rev. Lett. 99 1-4

[252] Lambert C H, Mangin S, Varaprasad B S D C S, Takahashi Y K, Hehn M, Cinchetti M, Malinowski G, Hono K, Fainman Y, Aeschlimann M and Fullerton E E 2014 All-optical control of ferromagnetic thin films and nanostructures Science 345 1337-40

[253] Manz S, Matsubara M, Lottermoser T, Büchi J, Iyama A, Kimura T, Meier D and Fiebig M 2016 Reversible optical switching of antiferromagnetism in TbMnO3 Nat. Photonics 10 653-6

[254] Liou Y De, Chiu Y Y, Hart R T, Kuo C Y, Huang Y L, Wu Y C, Chopdekar R V., Liu H J, Tanaka A, Chen C Te, Chang C F, Tjeng L H, Cao Y, Nagarajan V, Chu Y H, Chen Y C and Yang J C 2019 Deterministic optical control of room temperature multiferroicity in BiFeO3 thin films Nat. Mater. 18 580-7

[255] Yang M M and Alexe M 2018 Light-Induced Reversible Control of Ferroelectric Polarization in BiFeO3 Adv. Mater. 301704908

[256] Kundys B, Viret M, Colson D and Kundys D O 2010 Light-induced size changes in BiFeO3 crystals Nat. Mater. $9803-5$

[257] Vats G, Bai Y, Zhang D, Juuti J and Seidel J 2019 Optical Control of Ferroelectric Domains: Nanoscale Insight into Macroscopic Observations Adv. Opt. Mater. 71800858

[258] Rubio-Marcos F, Ochoa D A, Del Campo A, García M A, Castro G R, Fernández J F and García J E 2018 Reversible optical control of macroscopic polarization in ferroelectrics Nat. Photonics 12 2932

[259] Stoica V A, Laanait N, Dai C, Hong Z, Yuan Y, Zhang Z, Lei S, McCarter M R, Yadav A, Damodaran A R, Das S, Stone G A, Karapetrova J, Walko D A, Zhang X, Martin L W, Ramesh R, Chen L Q, Wen H, 
Gopalan V and Freeland J W 2019 Optical creation of a supercrystal with three-dimensional nanoscale periodicity Nat. Mater. 18 377-83

[260] Yang M, Kim D J and Alexe M 2018 Flexo-photovoltaic effect Science 907 904-7

[261] Chu K, Jang B K, Sung J H, Shin Y A, Lee E S, Song K, Lee J H, Woo C S, Kim S J, Choi S Y, Koo T Y, Kim Y H, Oh S H, Jo M H and Yang C H 2015 Enhancement of the anisotropic photocurrent in ferroelectric oxides by strain gradients Nat. Nanotechnol. $10972-9$

[262] Matzen S and Fusil S 2015 Domains and domain walls in multiferroics Comptes Rendus Phys. 16 227-40 